\title{
The common transcriptional subnetworks of the grape berry skin in the late stages of ripening
}

\author{
Ryan Ghan ${ }^{1}$, Juli Petereit ${ }^{2}$, Richard L. Tillett ${ }^{2}$, Karen A. Schlauch ${ }^{1,2}$, David Toubiana ${ }^{3}$, Aaron Fait ${ }^{4}$ \\ and Grant R. Cramer ${ }^{1 *}$ (D)
}

\begin{abstract}
Background: Wine grapes are important economically in many countries around the world. Defining the optimum time for grape harvest is a major challenge to the grower and winemaker. Berry skins are an important source of flavor, color and other quality traits in the ripening stage. Senescent-like processes such as chloroplast disorganization and cell death characterize the late ripening stage.

Results: To better understand the molecular and physiological processes involved in the late stages of berry ripening, RNA-seq analysis of the skins of seven wine grape cultivars (Cabernet Franc, Cabernet Sauvignon, Merlot, Pinot Noir, Chardonnay, Sauvignon Blanc and Semillon) was performed. RNA-seq analysis identified approximately 2000 common differentially expressed genes for all seven cultivars across four different berry sugar levels (20 to $26^{\circ} \mathrm{Brix}$ ). Network analyses, both a posteriori (standard) and a priori (gene co-expression network analysis), were used to elucidate transcriptional subnetworks and hub genes associated with traits in the berry skins of the late stages of berry ripening. These independent approaches revealed genes involved in photosynthesis, catabolism, and nucleotide metabolism. The transcript abundance of most photosynthetic genes declined with increasing sugar levels in the berries. The transcript abundance of other processes increased such as nucleic acid metabolism, chromosome organization and lipid catabolism. Weighted gene co-expression network analysis (WGCNA) identified 64 gene modules that were organized into 12 subnetworks of three modules or more and six higher order gene subnetworks. Some gene subnetworks were highly correlated with sugar levels and some subnetworks were highly enriched in the chloroplast and nucleus. The petal $\mathrm{R}$ package was utilized independently to construct a true small-world and scale-free complex gene co-expression network model. A subnetwork of 216 genes with the highest connectivity was elucidated, consistent with the module results from WGCNA. Hub genes in these subnetworks were identified including numerous members of the core circadian clock, RNA splicing, proteolysis and chromosome organization. An integrated model was constructed linking light sensing with alternative splicing, chromosome remodeling and the circadian clock.

(Continued on next page)
\end{abstract}

\footnotetext{
*Correspondence: cramer@unr.edu

1 Department of Biochemistry and Molecular Biology, University of Nevada,

Reno, NV 89557, USA

Full list of author information is available at the end of the article
} 
(Continued from previous page)

Conclusions: A common set of differentially expressed genes and gene subnetworks from seven different cultivars were examined in the skin of the late stages of grapevine berry ripening. A densely connected gene subnetwork was elucidated involving a complex interaction of berry senescent processes (autophagy), catabolism, the circadian clock, RNA splicing, proteolysis and epigenetic regulation. Hypotheses were induced from these data sets involving sugar accumulation, light, autophagy, epigenetic regulation, and fruit development. This work provides a better understanding of berry development and the transcriptional processes involved in the late stages of ripening.

Keywords: Circadian clock, Chromosome organization, Epigenetic modification, Fruit development, Grape berry, Network analysis, RNA-seq, Transcriptomics, Vitis vinifera $L$

\section{Background}

Fruits are specialized organs that encapsulate seeds. Botanically a fruit is the ripened ovary or carpel of a flower. Functionally it allows the development of and is a vehicle for the dispersal of seeds. There are different kinds of fruits. Fleshy fruit examples are grapes, apples and oranges; dry fruits include cereal grains and nuts. Both fleshy and dry fruits have similar regulatory subnetworks and developmental programs [1].

Grape berries are formed on flower clusters or inflorescences. Grape berry development and ripening involve complex physical and molecular changes [2], including color development, softening, volatile production, acid catabolism, and sugar accumulation. These processes at maturity or peak ripeness produce attractive signals for human, avian and other vectors of seed dispersal. Grape berries have many bioactive compounds, like polyphenols and carotenoids with bright colors and aromas that signal their edibility and health-related benefits [3, 4].

Grape berry color change and sugar accumulation are common metrics for ripeness. These processes change significantly at veraison, the start of the ripening stage in grapes. Sugar accumulation can vary with genotype and environment [2]. For example, higher temperatures lead to higher sugar accumulation in the berry at the optimum time of ripeness (as defined by the winemaker). Many times the winemaker will taste the berries to determine optimum flavor and time to harvest.

Nonetheless, sugar accumulation is a wine industry standard for assessing grape maturity. Wine grapes are typically harvested between 22 and $25{ }^{\circ}$ Brix. Other measures of fruit maturity exist (e.g. total acidity or the sugar to total acidity ratio), but the sugar level remains a simple and standard measurement of grape maturity in the wine industry. Measurement of sugar levels in grapes is an easy and accurate measurement; all that is needed is a simple refractometer that measures soluble solids ( ${ }^{\circ}$ Brix), which are essentially made up of glucose and fructose sugars. Wine grape berries typically can reach $25 \%$ sugar if allowed to ripen fully.
Higher concentrations can be achieved, but this is often due to dehydration of the berry. A recent paper characterizes in detail the effects of postharvest dehydration in these late stages of berry ripening [5], which are beyond the stages studied in the present study.

Sugars affect plant growth and development. Sugars provide energy for cellular respiration and transcriptionally signal and regulate gene activity, allowing the finetuning of fruit metabolism and development [6, 7]. Sugar can interact with hormones and the circadian clock to regulate gene expression [8]. In addition, sugar can induce senescence [9] and inhibit the expression of photosynthetic genes [10]. Some of the sugar sensors have been identified in plants [8] including hexose kinase (HXK1), SNF1 related protein kinase 1.1 (SnRK1.1) and target of rapamycin (TOR). A glucose phosphate transporter (GPT2) appears to be an important sugar sensor in the chloroplast [10].

Sugars can influence fruit development as well [11-13]. Sugar signaling has been linked to wheat grain development [13]. In strawberry, tomato [12], and grape [14], sugar influences the expression of the ABA-stressripening $(A S R)$ gene, a transcription factor, which when it is over-expressed or silenced, accelerates or delays fruit ripening, respectively [12]. In grapes, sugars enhance anthocyanin development and the expression of many genes, including the sugar sensor, HKT1 (High-affinity $\mathrm{K}$ (potassium) Transporter 1) [11, 15, 16].

Other factors can affect fruit ripening, such as epigenetic regulation and hormones $[1,2]$. An investigation of the tomato methylome showed epigenetic control of ripening that was tissue and developmentally specific at the breaker stage [17] when color begins to develop. Grape berry development is likely to be under epigenetic regulation as well $[18,19]$.

Hormonal regulation of fruit development is perhaps best studied in tomato [1]. A complex interplay of hormones are involved at different stages of fruit development. A number of grape studies indicate that different aspects of ripening are also under hormonal control 
by auxin, ethylene, abscisic acid and other hormones [2]. Most of these grape studies were focused on veraison.

A few studies have focused on the late ripening stages of grapes [2, 5, 20-22]. This stage of development represents a senescence-like phase preparing the fruit for seed dispersal with several degradative processes occurring including chloroplast disintegration [23, 24] and cell death [22]. A large number of genes and physiological processes appears to be operating including genes involved with ethylene signaling and flavor pathways [20] in the skin of Cabernet Sauvignon berries in the late stages of ripening over a range of ${ }^{\circ}$ Brix levels ( 22 to $37^{\circ} \mathrm{Brix}$ ). This present study focuses on a better definition of a core set of late ripening genes by expanding upon our previous findings in Cabernet Sauvignon. To better understand fruit ripening processes in this stage, four red-skinned and three whiteskinned grape cultivars were studied: Cabernet Franc, Cabernet Sauvignon, Merlot, Pinot Noir, Chardonnay, Sauvignon Blanc and Semillon, respectively. A narrower range of ${ }^{\circ}$ Brix levels was selected to restrict the set of candidate genes involved in berry skins to those genes that are expressed around the optimal sugar levels that flavor maturity develops; berry skins are the primary source of aroma, flavor and color in grapes [25].

Transcriptomic and gene network analyses can be used to infer active physiological processes in organisms. To derive and understand complex gene subnetworks (modules) in cells or whole organisms, scientists use gene coexpression network analysis approaches to identify highly connected gene subnetworks with highly enriched gene ontology (GO) categories [26-29]. WGCNA (Weighted Gene Co-expression Network Analysis) is one approach that was effectively used to identify highly connected hubs in gene subnetworks for Arabidopsis [30,31] and Vitis [32]. Another approach, petal [29], was developed recently to provide a truly scale-free and small-world network model for large-scale Omics analyses. A common set of transcriptional changes occurring in the late ripening stages for all cultivars was identified in this study using a standard (a posteriori) approach of gene mapping to known biochemical pathways and gene network (a priori) approaches. These approaches have elucidated multiple transcriptional processes of grape berry ripening at the mature stage and identified top hub genes in these gene subnetworks some of which involve autophagy, photosynthesis, chromosome organization and the circadian clock.

\section{Results}

Throughout September and October of 2012, whole berry clusters were harvested at the Valley Road Nevada Agricultural Experiment Station Experimental Vineyard. Seven grape cultivars were harvested: Cabernet Franc, Cabernet Sauvignon, Merlot, Pinot Noir, Chardonnay, Sauvignon Blanc and Semillon. Individual berry skins were separated immediately from the whole berry and the individual sugar ( $\left.{ }^{\circ} \mathrm{Brix}\right)$ level of the berries was determined. RNA-seq profiling of transcript abundance during the late stages of development was then conducted on berry skins at different sugar levels (see Methods for details). Prior to signal filtering, there were 27,926 expressed genes out of 29,971 annotated genes in the V1 reference genome (Additional file 1). Independent filtering of lowly expressed genes by minimum counts mapped (see Methods) reduced the count to 16,606 genes for downstream analysis (Additional file 2).

A principal components analysis (PCA) was performed (Fig. 1) to validate sample uniformity and investigate the degree of separation between cultivar and ${ }^{\circ}$ Brix effects. Cultivars were distinctly separated on the 1 st principal component explaining $21.8 \%$ of the variance, with red and white cultivars segregating together and away from one another. ${ }^{\circ}$ Brix levels segregated along the 2nd principal component explaining $21.4 \%$ of the variance, in some cases distinctly from one another (e.g. Merlot at $20{ }^{\circ}$ Brix, Semillon and Chardonnay both at $26^{\circ}$ Brix). The cultivars separated in a similar pattern as in a previous study [33], with Pinot Noir again segregating between red and white cultivars.

While there were many transcripts changing that were different for different cultivars, in this presentation of the results we focus on the common changes in transcript abundance amongst the seven grape cultivars at different ${ }^{\circ}$ Brix levels. We define a common differentially expressed gene (DEG) as the gene's transcript abundance changing significantly in at least one Brix comparison of all possible comparisons (e.g. 24 vs 22,26 vs 20,24 vs 22 , etc.) AND this had to occur for at least one comparison in every cultivar. We identified 2108 common differentially expressed genes (DEGs) using a series of contrasts between each ${ }^{\circ}$ Brix level (Additional files 3 and 4).

In the following analyses, we utilized the DEGs to conduct a "standard" transcriptomic analysis approach, followed by two different gene co-expression network analysis approaches. In the first approach (standard network analysis), gene set enrichment of gene ontologies (GO) was utilized to identify "genes of interest". They were then mapped to known physiological and biochemical pathways (a posteriori approach) to gain further insight. In the second approach, we applied two different gene co-expression network analyses, WGCNA and petal, to the set of 16,606 filtered and quality-controlled transcripts (a priori approach) and evaluated some of the gene subnetworks and hubs identified.

\section{Standard transcriptomic analysis Gene ontology enrichment analysis}

Enriched GO categories for the 2108 DEGs for the common genes of all cultivars (the ${ }^{\circ} \mathrm{Brix}$ effect) were identified 


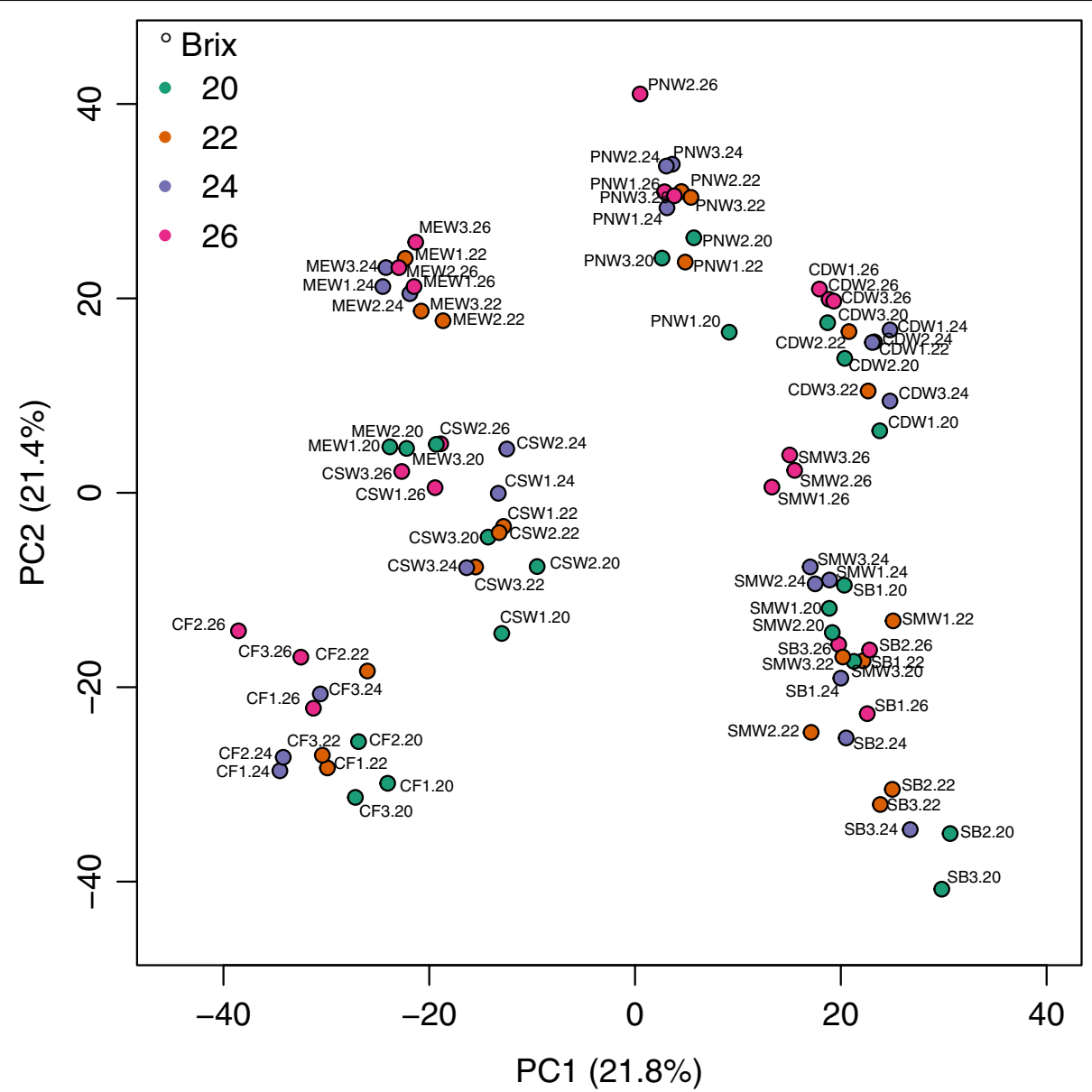

Fig. 1 A PCA plot of berry skin samples according to their normalized counts per million. The first $(P C 1)$ and second (PC2) components are represented. Samples corresponding to three biological replicates from four ${ }^{\circ}$ Brix levels were analyzed. ${ }^{\circ}$ Brix levels are colored across cultivars. Sample abbreviations represent the cultivar, replicate number and Brix level, respectively. Cultivar abbreviations are Cabernet Franc (CF), Sauvignon Blanc (SB), Cabernet Sauvignon (CS), Merlot (ME), Pinot Noir (PN), Chardonnay (CD) and Semillon (SM)

(Additional file 5) and were depicted in a GO network (Additional file 6). The color and size of the circles signify the level of enrichment and the number of genes in each set, respectively. Approximately $400 \mathrm{GO}$ categories were overrepresented after correcting for multiple hypothesis testing (Additional file 5). Mapping the DEGs on the cellular overview of the Vitiscyc [34] webpage showed that these transcripts were widespread across most biochemical pathways (data not shown). These results indicated that grape berry ripening in the late stages was broad and complex across many biochemical pathways.

Some of the top overrepresented GO categories included membrane, regulation of hormone levels, catabolic process and response to abiotic stress. Other interesting GO categories included developmental process, response to light, response to monosaccharide, nitrogen metabolism, RNA processing and chloroplast. Many GO categories related to flavor development were overrepresented (e.g. fatty acid catabolism, amino acid metabolism, alcohol metabolism and isoprenoid metabolism).
Of the 2108 DEGs, approximately half of the transcripts increased and the other half decreased with increasing ${ }^{\circ}$ Brix level (Fig. 2). Gene set enrichment was performed for the gene ontologies of the top 500 DEGs that increased and decreased the most (the difference in transcript abundance between $20^{\circ}$ and $26^{\circ}$ Brix) to determine the biological processes involved. The top three enriched GO categories (from a total of 105) for the increasing DEGs were nucleic acid metabolism, MCM (minichromosome maintenance) complex and chromosome organization (Additional file 7). Additional interesting categories that were highly enriched were DNA methylation and lipid catabolism. The top three GO categories (from a total of 197) for the decreasing DEGs were membrane, cell wall and photosystem (Additional file 8). Other interesting categories included regulation of hormone levels and pigment accumulation.

Examples of three of the top genes with increased and decreased transcript abundance with increasing ${ }^{\circ}$ Brix level include a MYB transcription factor, a LOB domain 


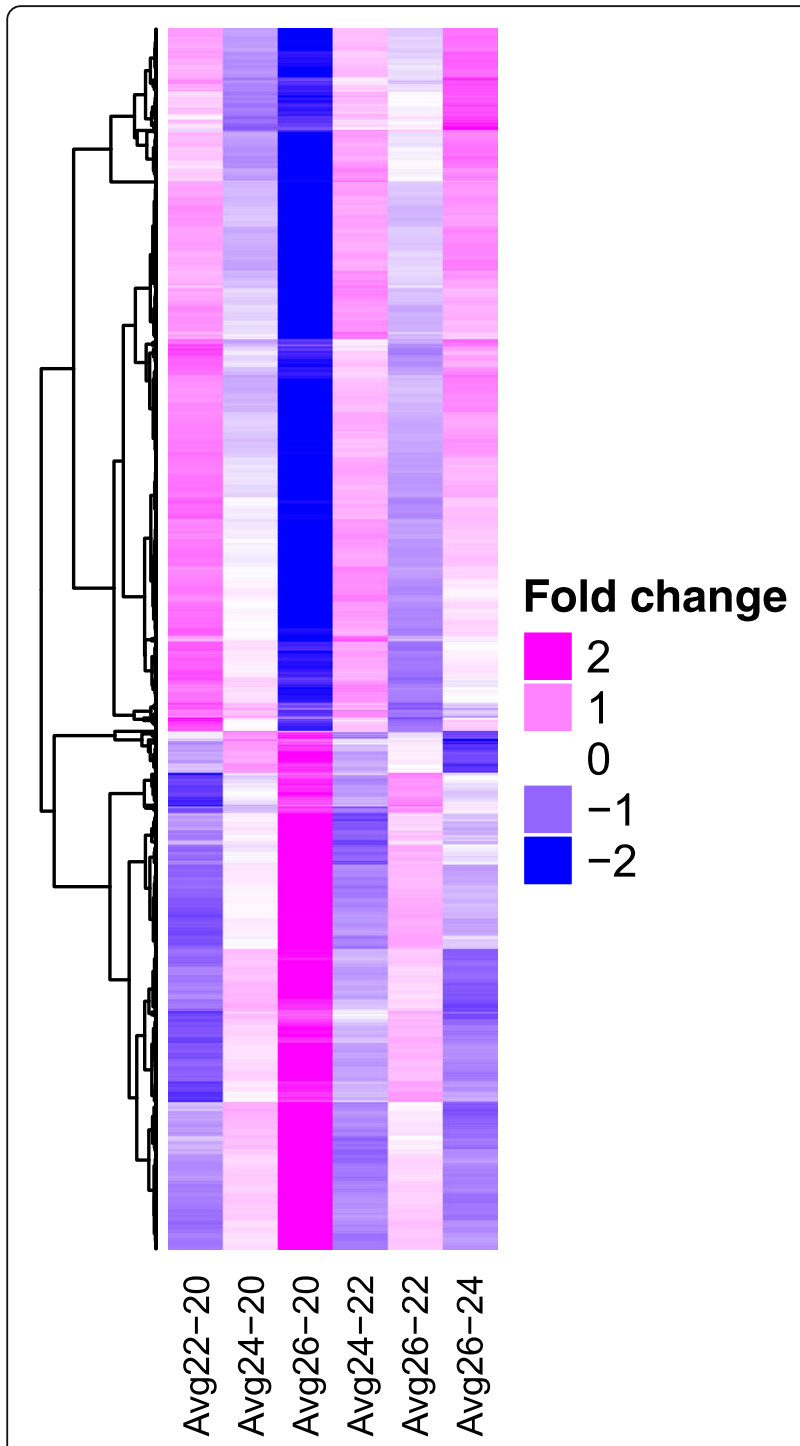

Fig. 2 A heat map representing the difference in log2 expression of the 2108 DEGs with different ${ }^{\circ}$ Brix contrasts. Individual ratios for the six contrasts were computed for each cultivar and then averaged

protein, a ubiquitinase, an expansin, a bifunctional lipid transfer protein and a pectin lyase (Fig. 3).

\section{Chromosome organization and regulation of transcription}

Gene ontology enrichment analysis indicated that chromosome related events (chromosome organization, DNA and histone methylation, etc.) were significantly modified in the late stages of ripening (Additional file 5). Chromatin remodeling was recently discovered to be very important in regulating tomato fruit ripening [17]. Many genes related to chromatin silencing or chromosome organization that negatively regulate gene transcription were associated with increasing ${ }^{\circ}$ Brix. These included histone methyltransferases and a number of sucrose non-fermentable 2 (SNF2) transcripts. SNF2 domain-containing proteins participate in epigenetic regulation of gene transcription to control development in plants and other organisms [35]. For example, the transcript abundance of Photoperiod Independent Early Flowering 1 (PIE1; VIT_08s0007g06370) whose protein contains helicase and SNF2 domains, was increased with ${ }^{\circ}$ Brix level (Fig. 4). Similarly, the transcript abundance of the transcription factor VviDDM1 (Decrease in DNA Methylation 1; VIT_04s0023g01610) also peaked at $26^{\circ}$ Brix. DDM1 belongs to the Lsh subfamily of SNF2 domain proteins $[35,36]$. The transcript abundance of another TF, methyl-CPG-binding Domain 9-like (MBD9; VIT_14s0066g01450) also significantly increased with Brix. And finally, a minichromosome maintenance family protein (MCM; VIT_07s0005g01430) increased with increasing ${ }^{\circ}$ Brix.

\section{Regulation of hormone level genes}

Genes involved in the regulation of hormones were also significantly enriched. The regulation of several different hormone pathways was represented in this group (Fig. 5). A protein kinase receptor, HSL1 (HAESAlike 1; VIT_10s0003g00330) is one of the most significantly reduced DEGs with increasing ${ }^{\circ}$ Brix. It interacts with an abscission signaling peptide to inhibit seed maturation [37, 38]. Its expression is dependent on sugar levels and it also interacts with sugar-inducible and ABAregulated genes $[39,40]$. A reduction in transcript abundance of VviHSL1 may contribute to seed maturation.

Other genes with decreasing transcript abundance (Fig. 5) included Non-Phototropic Hypocotyl 3 (NPH3; VIT_03s0038g00270), Auxin Response Factor 3 (ARF3; VIT_10s0003g00420), and Oxophytodienoate-Reductase 3 (OPR3; VIT_11s0016g01230). NPH3 regulates auxin efflux carriers [41] and OPR3 is involved in jasmonate biosynthesis [42].

\section{Light responsive and photosynthetic genes}

Many blue-light responsive genes, including Zeitlupe (VviZTL; VIT_11s0052g00730) and XAP5 Circadian Timekeeper (VviXCT; VIT_03s0038g01810) had an increasing response to ${ }^{\circ} \mathrm{Brix}$ level. These genes are known to measure day length and adjust the circadian clock. The transcript abundance of a Constans-Like 4 gene (VviCOL4, VIT_04s0008g07340) was increased significantly with ${ }^{\circ}$ Brix. Constans-like genes were first identified in flowering and are important sensors of day length and light-driven redox signaling [43]. Constans-like 13 (VIT_07s0104g01360) belongs to Group III of CO-like TFs [44, 45]. Almada et al. [44] reported both spatial and temporal expression patterns for the VviCOL1, with a reduction of expression in maturing berries, a pattern seen in all cultivars.

Nearly all of the transcripts for photosynthetic DEGs were decreasing with increasing ${ }^{\circ}$ Brix level, such as 


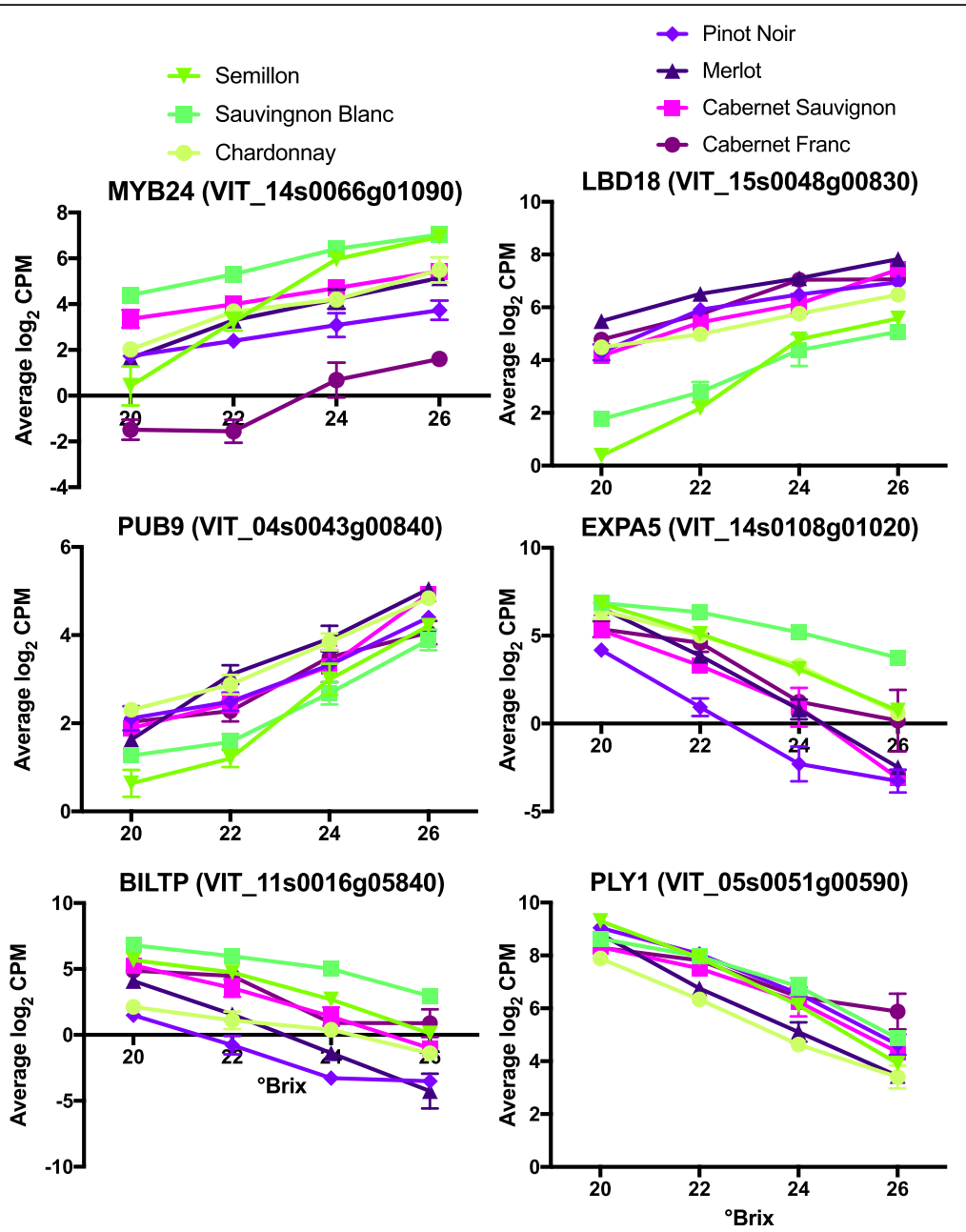

Fig. 3 The transcript abundance of some of the top DEGs. Data shown are the means $\pm S E ; n=3$. Error bars not shown are smaller than the symbol. The symbol legend is displayed in the figure

Cytochrome C6A (VIT_01s0011g01850) or light harvesting complex II type I CAB-1 (VIT_10s0003g02890). The gene expression of some of these photosystem genes appears to be completely shutting down. Only two transcripts associated with photosynthesis were increasing in expression: a pentatricopeptide repeat-containing (PPR) protein (VIT_03s0063g00900) and ferritin (VIT_08s0058g00440). These results support the hypothesis that chloroplasts are becoming nonfunctional for photosynthesis or possibly even degraded. Chloroplasts are also the location for isoprenoid, carotenoid and terpenoid metabolism, and thus a source of important volatiles and aromas [46].

\section{Gene co-expression network analyses WGCNA}

WGCNA was applied to the entire set of transcripts to systematically and globally identify highly-connected gene subnetworks or module eigengenes (MEs), which are the first right-singular vectors of the standardized module expression data. WGCNA was also used to identify highly correlated hub genes within these modules. Results from the WGCNA $\mathrm{R}$ package hierarchical clustering function confirmed the PCA results that the transcript abundance of all quality-controlled transcripts of the berry samples separated very well according to cultivar and ${ }^{\circ}$ Brix effects (Additional file 9). WGCNA produces module memberships with highly enriched GO categories with important biological meaning $[26,32]$. WGCNA defines modules as clusters of gene nodes with high topological overlap, which means that members of a given module share a greater number of connections with other members of the module than with genes outside the module [27]. The gene network had near scale-free topology (Additional file 10) with a number of highly connected hub gene nodes. Extensive branches (clusters of transcripts forming modules) can be observed in the gene dendrogram produced using average linkage hierarchical clustering (Additional file 9). Utilizing the WGCNA R package, 64 

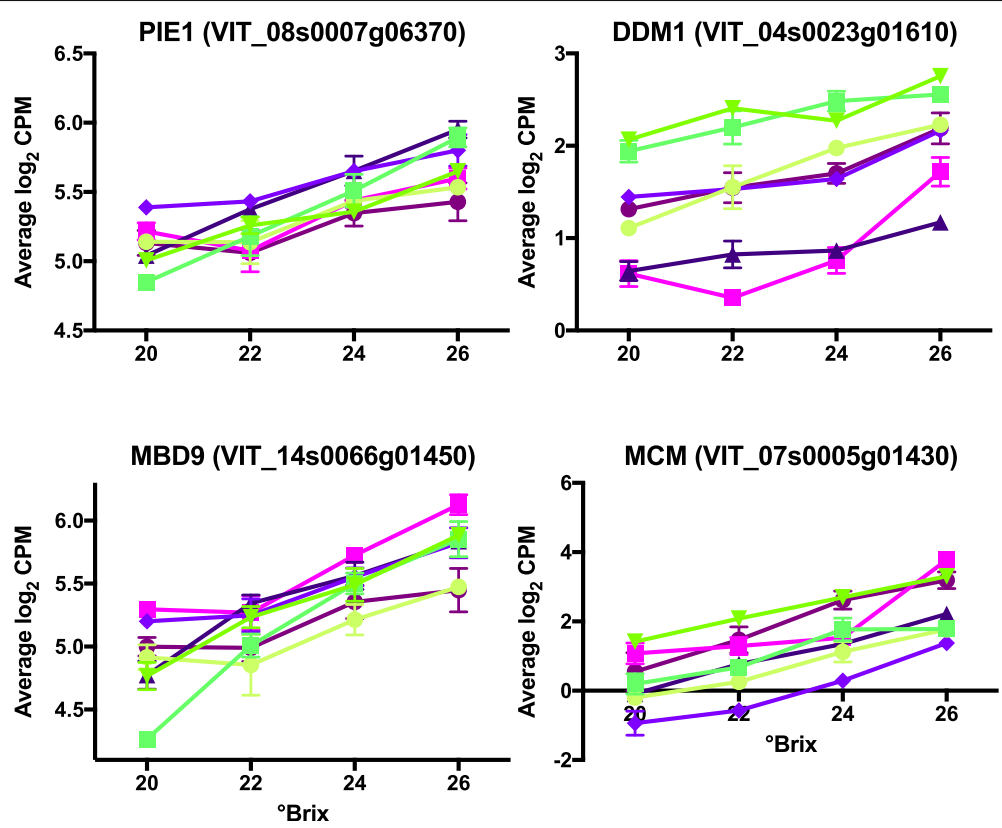

Fig. 4 Expression profiles of some genes involved in chromosome organization. Data shown are the means $\pm S E ; n=3$. Error bars not shown are smaller than the symbol. Symbol legend is the same as in Fig. 3

modules were identified by applying the cuttreeDynamic function with a minimum membership of 30 genes. Modules were merged based on a threshold of 0.25 (see Methods for details and the colored blocks in the merged dynamic in Additional file 9). The color grey is reserved for genes not belonging to any module, thus the grey module (MEgrey) does not present a real module.
A module eigengene was calculated for each module and correlated with the berry traits: ${ }^{\circ}$ Brix level, cultivar, red or white grapes (Fig. 6). Each square in the figure table was colored if it was statistically significant and labeled with two numbers; the upper number is the correlation coefficient and the lower number is the $p$-value. Some modules were significantly correlated with specific traits. Here we focus on modules most correlated with

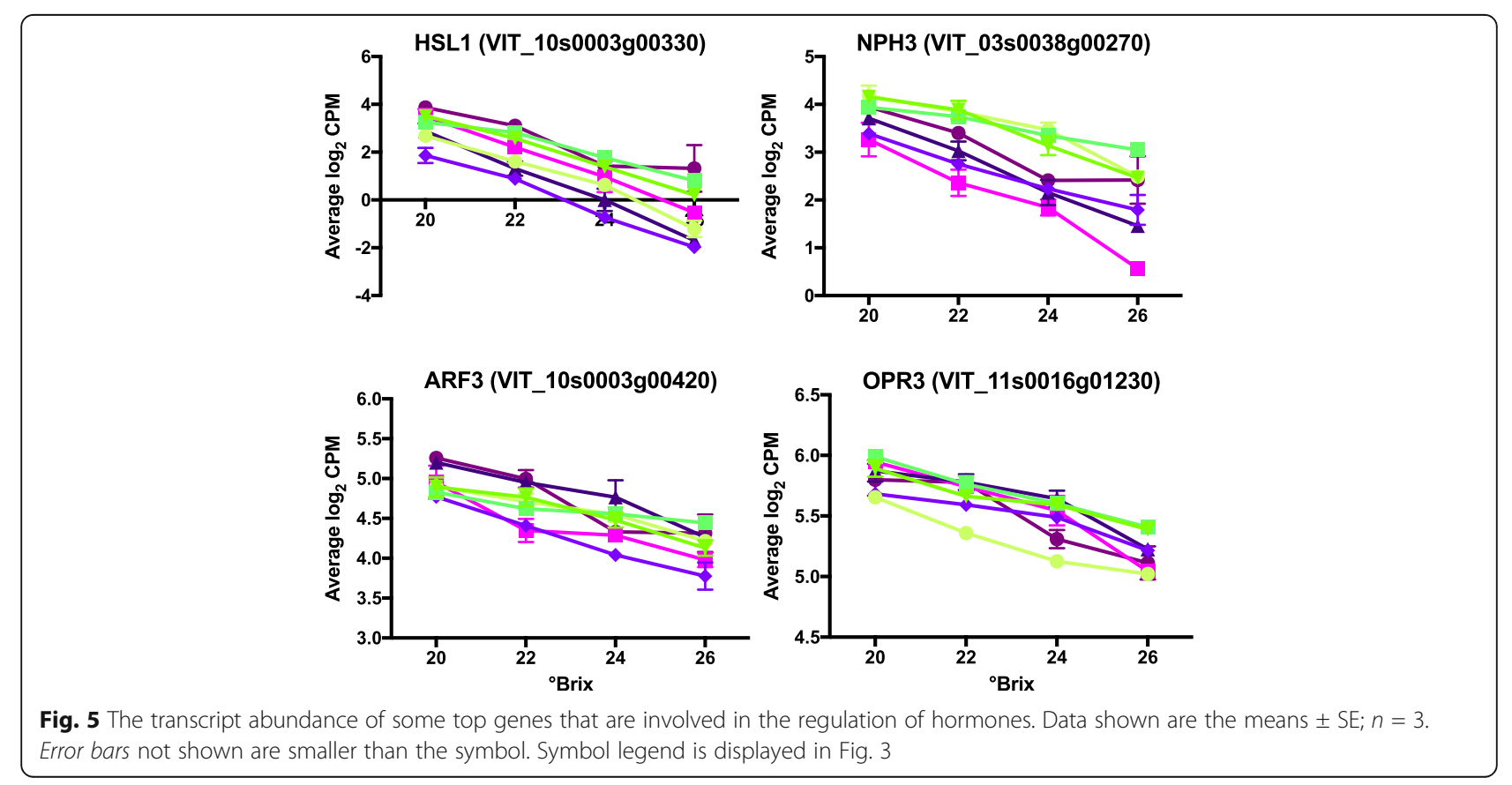


the ${ }^{\circ}$ Brix levels. The orangered3, turquoise, antiquewhite 1 and coral 2 were the most significantly correlated modules with an increasing gene expression trend with increasing ${ }^{\circ}$ Brix (Fig. 6). The darkgrey, navahowhite, darkseagreen 3 and lavenderblush 3 modules were the most significantly correlated modules with a decreasing gene expression trend with increasing ${ }^{\circ}$ Brix.

Module membership (kME or the module eigengene connectivity) was calculated for each gene for each module (Additional file 11). A kME value of 1 indicates perfect correlation with the module eigengene and the higher the kME of a gene the higher its connectivity within the module. Genes with a high kME are considered hub genes. GO category enrichment was determined with the top 500 genes of each module (data not presented).

Major details of all 64 modules were summarized (Additional file 12), including the top hub gene and GO categories highly enriched in each module. Half of the 64 modules were significantly correlated with ${ }^{\circ}$ Brix (Additional file 12). Many of these modules were enriched with many GO categories including chloroplast, ribosome, cytoplasm, nucleus, photosynthesis, translation, nucleic acid metabolism, phenylpropanoid biosynthesis, defense responses, etc.

Module eigengenes for all 64 modules were correlated with each other to elucidate the relationships between modules (Fig. 7). Hierarchical clustering revealed a complex network order; 6 higher order subnetworks (A to F) could be subdivided into what we call "module subnetworks" (1 to 12). The 12 module subnetworks were identified with a minimum set of three modules (Additional file 12, Fig. 7).

The higher order subnetworks, A, B, and C, all were highly enriched in chloroplast, cytoplasm, photosynthesis and translation GO categories (Additional files 11 and 12). They were mostly associated with higher expression in white grape varieties, but there were a significant number of modules that had no differences between red and white grapes.

Higher order subnetwork D was enriched in the nucleus, nucleotide binding and gene expression. Higher order subnetwork $\mathrm{E}$ was not enriched in any particular cellular compartment but was enriched in phenylpropanoid and aromatic compound metabolism GO categories. Higher order subnetwork $\mathrm{F}$ was enriched in the golgi apparatus and transport processes. These latter higher order subnetworks (D-F) were mostly associated with higher expression in red grape varieties, but there were a significant number of modules that had no differences between red and white grapes (Additional file 12); these represent common gene subnetworks for all cultivars.

Some module subnetworks were large and complex, consisting of 8 or 9 modules (Additional file 12, Fig. 7); they formed a subnetwork within a larger subnetwork. For example, the Group 3 module subnetwork consisted of modules involved in protein folding, translation, chloroplast and response to light. Only 3 of the 8 modules in this subnetwork were correlated (negatively) with increasing ${ }^{\circ}$ Brix, the rest were more correlated with different genotypes (Additional file 12, Fig. 6).

The Group 2 module subnetwork consisted of 6 modules and was similar to Group 3; the module clustering in the module dendrogram indicated that the subnetworks formed into a larger branch or subnetwork. Five of the six modules in Group 2 negatively correlated with increasing ${ }^{\circ}$ Brix. These modules had gene ontologies involving the chloroplast, translation, and photosynthesis and were similar to those in Group 3.

In summary, the WGCNA network of the grape berry skin at the late ripening stages could be divided into 6 higher order subnetworks and 12 module subnetworks. These subnetworks were enriched in GO categories involving cellular compartmentation (e.g. chloroplast and nucleus) and major metabolic processes (e.g. photosynthesis, translation, and phenylpropanoid metabolism). In the next section we will focus on details in the two largest gene modules identified by WGCNA that were correlated with ${ }^{\circ}$ Brix levels.

\section{Turquoise module had the largest positive correlation with Brix}

The turquoise module was highly correlated with three other modules: orangered3, antiquewhite1 and coral2 (Fig. 7). These four modules formed the core of the Group 6 module subnetwork (Fig. 7). There were many common GO categories amongst these modules including nucleic acid and protein binding (Additional file 12). Interspersed within the top kMEs were many of the core circadian clock genes (Additional file 11).

The turquoise module eigengene had the highest significant correlation of 0.69 ( $p$-value $=4 \mathrm{e}-10$; Additional file 12; Fig. 6) with $26^{\circ}$ Brix. The turquoise module was the largest most connected module with 355 transcripts having a $0.80 \mathrm{kME}$ or higher. Transcript abundance in this module increased with ${ }^{\circ} \mathrm{Brix}$ level. The response was generally common for all varieties $(p$-value $>0.1$ for red vs white grape and all varieties except Cabernet Sauvignon). The top hub was VIT_13s0067g03760, a pre-mRNA-splicing factor 3 protein, with a kME of 0.96 . The protein of this gene is required for RNA-directed methylation of DNA [47].

There were 337 GO categories overrepresented in the top 500 genes in this module (data not shown). Highly enriched GO categories of the top 500 turquoise module genes included nucleic acid binding ( $p$-value $=1.74 \mathrm{e}-17)$, nucleus (2.86e-15), mRNA metabolic process $(9.28 \mathrm{e}-17)$, and protein binding (1.11e-11). Other interesting GO 


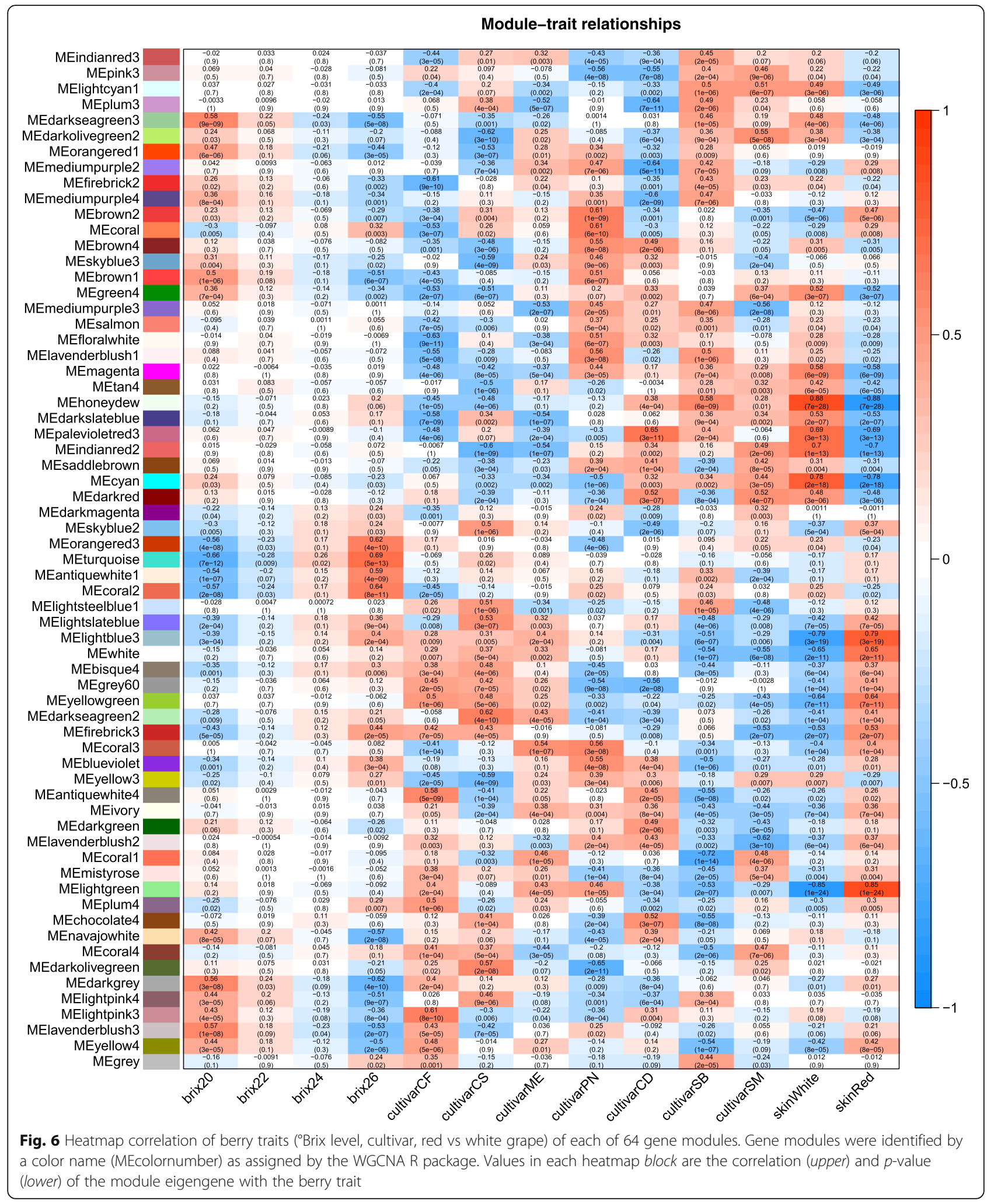

categories included RNA processing, epigenetic regulation of gene expression, post-embryogenic development, response to red or far red light and autophagy.
The top 100 genes of this module were highly enriched in specific categories that may be interacting with each other; 32 were involved in RNA processing, 22 were 


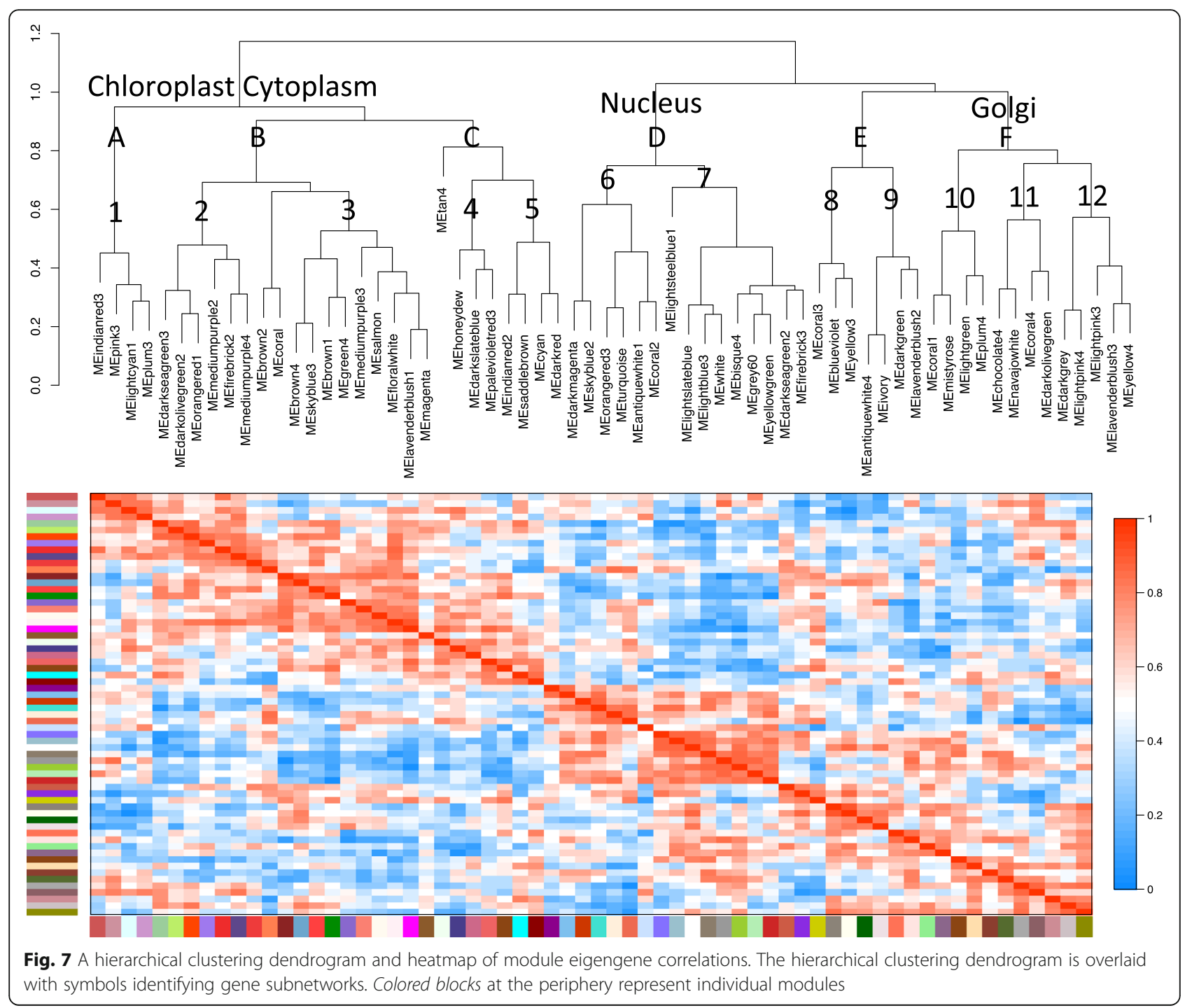

involved in chromosome organization, 12 were involved in proteolysis, 9 were transcription factors, 2 were involved in the circadian clock or circadian rhythm and 2 were involved in autophagy (See Additional file 13 for color highlights and a list of genes in each category). Many of these processes are intimately connected to the core circadian clock (see Discussion). These genes represented 79 of the total top 100 hubs, indicating very high enrichment of these $\mathrm{GO}$ categories in the top 100 genes.

Some of these top $100 \mathrm{kME}$ genes were involved in more than one category. For example, CBF1-interacting corepressor CIR domain containing protein (VIT_02s0087 g00830; hub rank \#43), Enhancer of polycomb-like transcription factor protein (VIT_04s0008g04370; hub \#67), Microrchidia 4 (MORC4; VIT_17s0000g00910; hub \#75), RNA Polymerase II large subunit (VIT_18s0001g00860; hub \#89), High Mobility Group (HMG; VIT_14s0108g00
040; hub \#92) are involved in both RNA processing and epigenetic regulation; methyl-CPG-binding domain 9 (MBD9; VIT_14s0066g01450; hub \#25) and DDB1-CUL4 associated factor 1 (VIT_04s0008g03060, hub \#9) are involved in RNA processing and protein ubiquitination.

The turquoise module was highly connected to the circadian clock and other light regulated genes; the module contained the largest number of circadian clock and light-regulated genes (16) in the top 500 kMEs (positively correlated) than any other module (Additional file 11); it also had 5 circadian clock and light-regulated genes in the bottom 500 kMEs (negatively correlated), inferring that these genes may have been negatively regulated or repressed by the same factors that positively regulated the genes within the turquoise module. The light-regulated gene from the turquoise module with the highest $\mathrm{kME}$ was COP1-interacting protein 4.1 (CIP4; VIT_01s0137g00190; 0.91 kME, hub \#37). CIP4 
was highly connected in 3 similar modules: its hub rank was equal to \#37, \#214, and \#282 for turquoise, orangered3, and coral 2 modules, respectively. CIP4 is a transcriptional activator that promotes photomorphogenesis [48]. It appears to act downstream of most photoreceptors and Constitutive Photomorphogenic 1 (COP1). Photoreceptors can inhibit COP1 and thus activate CIP4.

The next highest hub in this group was Time for Coffee (TIC; VIT_05s0020g03150; 0.89 kME; hub \#55); it is the circadian clock gene with the highest connectivity. It interacts with $L H Y$ (Late Elongated Hypocotyl) and PRR9 (Pseudo-Response Regulator 9) in the core circadian clock [49]. ZTL, also known as Adagio Protein 1 (VIT_11s0052g00730), was the next most connected circadian clock hub gene, having a kME of 0.87 and had a rank of 101 in the module.

$E L F 3$, is part of the evening complex (ELF3, Early Flowering 4 (ELF4) and Phytoclock 1 (PCL1)) and appears to be a key regulatory hub for the circadian clock that modulates light signals [49]. The gene expression trends for Far-Red Impaired Response 1 (FAR1) and other FAR1-related genes, ELF3, ZTL, Topless (TPL), and COP1 within our data set were very similar and indicate a possible light signal sensing cascade/cycle involving FAR1 (or a FAR1- related sequence protein), PHYB (phytochrome B), ZTL, ELF3, COP1 and TPL.

$T P L$ was another interesting gene from the circadian clock core in this subnetwork of increasing gene expression; it forms a complex with PRR9 and HDA6, a histone deacetylase [50] that positively regulates chromosome compaction. TPL is a broad repressor of many genes and down-regulates the early morning genes as a co-repressor with PRR9, which binds to the promoters of the morning genes, CCA1 (Circadian Clock Associated 1) and $L H Y$, inhibiting their expression. Increasing TPL shortens the day phase (and lengthens the night phase) as in short days. It also interacts directly with EMF1 (Embryonic Flower 1) and WRKY32 [51]; both of these genes were within the top 100 kMEs of this module. EMF1 (VIT_04s0008g03660; $0.88 \mathrm{kME}$; hub \#74) is part of a Polycomb group (PcG) complex (chromatin modification mediating transcriptional repression) and regulates flowering by repressing Flowering Locus $T$ (FT) expression; it helps to synchronize environmental cues and is a vascular signal.

Another top hub, Early Flowering in Short Days (EFS; VIT_18s0001g01700; 0.93, 17) is a histone lysine Nmethyltransferase required specifically for the trimethylation of H3-K4 in Flowering Locus C (FLC) chromatin. It also affects carotenoid biosynthesis genes, and light and carbon responsive genes. EFS methylates $L H Y$ in Arabidopsis [52]. Other top hub genes were histone ubiquitin ligases (HUBs) involved in protein processing and post-embryonic development. Monoubiquination of histones by HUBs stimulates gene expression [53]. Thus, there is a strong representation of key components of the circadian clock in this gene module.

There were 4 autophagy genes in the top 500 kMEs of the turquoise module: homolog of yeast autophagy $18 \mathrm{G}$ (ATG18g), autophagy 9 (APG9), autophagy-related 11 (ATG11) and autophagy 2 (ATG2). In particular, 3 of the 4 are part of the APG9 cycling. ATG18g (hub \#20) was the most highly connected autophagy hub followed by APG9 (hub \# 122). Autophagy is an important part of the senescence process $[54,55]$.

\section{Darkseagreen3 module had the largest negative correlation with ${ }^{\circ}$ Brix}

The darkseagreen 3 module was part of the Group 2 module subnetwork and thus a part of one of the largest subnetworks elucidated in the late stages of berry ripening. The darkseagreen 3 module eigengene had a significant correlation of -0.55 ( $p$-value $=5 \mathrm{e}-08)$ with $26^{\circ}$ Brix. It was the second largest most connected module with 342 transcripts having a $0.80 \mathrm{kME}$ or higher. The transcript abundance of gene members in this module decreased with increasing ${ }^{\circ}$ Brix level. This decreasing response was generally common for all varieties, but there was higher transcript abundance in white grape skins. The top hub was VIT_09s0002g04360, a CURvature Thylakoid1 protein (CURT1), with a kME of 0.96, a membrane phosphoprotein responsible for curvature at the grana margins [56].

There were $422 \mathrm{GO}$ categories overrepresented. The top GO categories of the top 500 kMEs were chloroplast $(2.95 \mathrm{e}-47)$, thylakoid $(6.29 \mathrm{e}-46)$, photosynthesis (3.59e-32), and cytoplasm (4.27e-29). Other interesting GO categories included isopentenyl diphosphate biosynthetic process, mevalonate-independent pathway, alcohol metabolic process, monosaccharide metabolic process, lipid biosynthesis, and cysteine biosynthesis.

There were 7 and 8 circadian-related and light regulated genes in the top and bottom $500 \mathrm{kMEs}$, respectively. ELF4, part of the evening complex, was the top circadian clock gene at \#106, another circadian rhythm gene was Plastid Transcriptionally Active 16 (PTAC16; VIT_06s0004g05230; $0.86 \mathrm{kME})$ at \#113, Transcription factor TCP domain protein 7 (TCP7; VIT_10s0042g00170; hub \#180), Accumulation and Replication of Chloroplast 5 (ARC5; VIT_12s005 5g00490; hub \#221), Light-Inducible And Clock-Regulated 2 (LNK2; VIT_13s0139g00360; hub \#223), and Regulator of Chromosome Condensation (RCC1; VIT_07s0031g02560; hub \#455).

Some of the top negatively correlated circadian clock genes included VviELF3, VviZTL, VviSKIP (SNW/SKI-Interacting Protein; VIT_11s0016g03290), VviTIC (VIT_05s00 20g03150), VviRVE1 (ReVeillE 1; VIT_04s0079g00410) and VviFRS5 (FAR1-Related Sequence 5; VIT_03s0038g04140). These genes were all positively correlated hubs in the 
turquoise module and indicated a coordinated regulation between the turquoise and darkseagreen 3 module.

\section{Petal analysis}

To provide another independent approach for gene coexpression analysis, petal analysis was performed on the same set of 16,606 filtered and quality-controlled genes. Petal constructs models based on seven different Spearman correlation thresholds and calculates multiple topological network measures to automatically determine the 'best' possible network model for this dataset meeting scale-free and small-world characteristics [29]. The final model used for down-stream analysis (module and hub identification) is constructed based on a Spearman correlation value of 0.703 and includes 15,092 of the 16,606 original genes (Additional file 14). Here we define hub genes by the top $5 \%$ of connected genes within the final network model corresponding to 751 genes; their connectivity (k) ranged between 395 and 788. All maximal largest cliques within the hub subnetwork were extracted, resulting in 90 cliques (completely connected subnetworks) with 89 genes each. The intersection and union of these largest cliques include 74 and 105 genes, respectively. The union is a highly intra-connected subnetwork of 105 genes with a density of 0.99 (i.e., 46 edges (links) are missing to make a clique).

To build a larger subnetwork, the common immediate neighbors of the 105 hub genes were combined into what we define as a hub module; this resulted in a module of 216 genes with density of 0.85 (Additional file 15). The genes in this hub module (216 genes, Additional file 15) were also represented in the WGCNA turquoise module. Both approaches independently identified gene modules with highly connected hub nodes, although WGCNA's turquoise module included more genes, its intraconnectivity was much lower, indicating that the gene expression profiles within the WGCNA turquoise module exhibited greater variability compared to petal's hub module. There was general agreement of the order of hubs in petal with the kME values of the hubs in the turquoise module (Additional file 15). A true comparison is difficult, because WGCNA's kME values are local hub measures, whereas petal defines hubs based upon the entire small-world and scale-free network model. Thus, we can conclude that the two gene co-expression network approaches consistently identified a list of highly connected hubs in a common network of all grape cultivars of the late stages of grape berry ripening.

\section{Integration of WGCNA module subnetworks with the circadian clock in berry skins}

Several other WGCNA modules had circadian clock genes in the top (most positive) or lower (most negative) 500 kMEs. To better understand the role of the circadian clock in fruit ripening, an integrated circadian clock model was constructed relating known circadian clock genes with the grape berry skin gene modules in which they had the highest correlation (Fig. 8). The model integrated both blue and red light receptors (identified with red and blue lightning bolts) as well as genes involved in biochemical processes regulating the clock. These processes included light sensing, proteolysis, alternative splicing and chromatin remodeling. The model depicted a complex interplay of these processes and the large number of gene modules that were affected. Lines in the model represent known interactions between genes; red arrows are positive interactions, black lines are negative interactions, and blue lines indicate direct physical interactions but the direction, positive or negative, is unknown. No lines indicate that there are no known interactions at this time.

From this model it is hypothesized that light signals (in this case a shortening of the day length with a daily sunrise occurring later each day as time passes in Autumn and ${ }^{\circ}$ Brix increases in the berry) drive the resetting of the clock through the photoreceptors. These in turn affect a series of positive and negative feedback loops in the model.

More subtle associations in the model can be perceived through the module correlations. The two modules participating the most in the clock were the turquoise (14 members) and cyan (8 members) modules. ZTL, FRS5 and FRS6 photoreceptors were part of the turquoise module. The Cryptochrome 3 (CRY3) photoreceptor was part of the cyan module. There is no direct interaction evidence of these photoreceptors to the core clock components, but their expression was highly correlated with some clock components (e.g. HY5 (Elongated Hypocotyl 5), ELF3, COP1, PHYE (Phytochrome E), SPA3 (suppressor of phytochrome A-related 3, etc.) indicating some sort of linkage. In other cases, module associations were more obvious. For example, ZTL, a blue light photoreceptor, has a number of known interactions with clock components, including the inhibition of TOC1. LNK2 has direct positive effects on ELF4.

At a higher gene subnetwork level, module subnetwork 6 was largely represented by circadian clock components in the turquoise, coral2, antiquewhite1 and skyblue2 modules. Again, these modules were enriched in the nucleus, RNA splicing, chromosome organization, epigenetic regulation, and ubiquitination. The circadian clock components of the darkseagreen3, cyan, firebrick3 and lavenderblush3 modules were each part of the module subnetworks 2, 5, 7 and 12 , respectively. These subnetworks were enriched in the chloroplast and the cytoplasm categories.

Thus, this model depicted different genes (or proteins) within the circadian clock that had direct positive and negative interactions with other genes or proteins in 


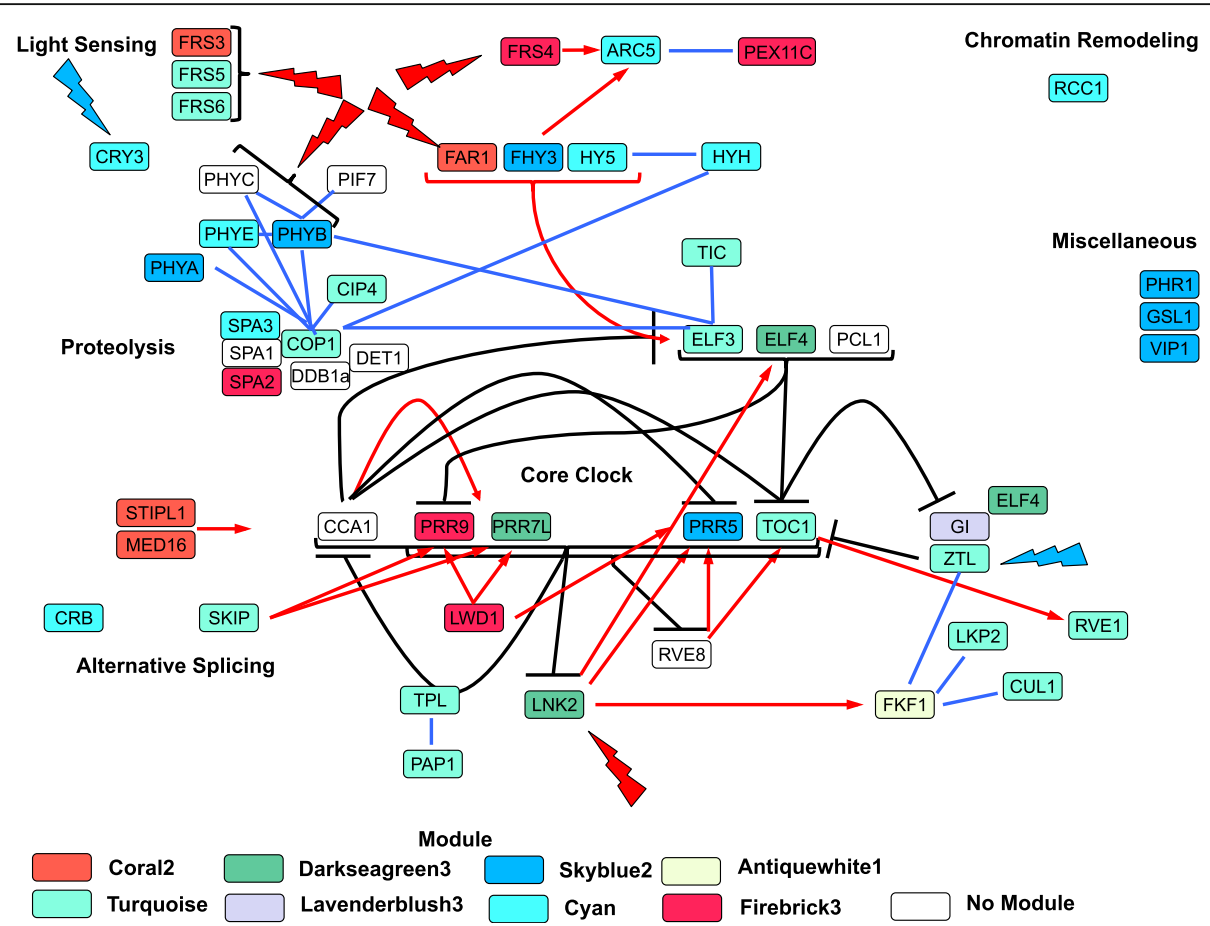

Fig. 8 An integrated circadian clock model with gene modules of grape berry skins in the late ripening stages. Colored symbols indicate genes within that particular module. Light sensing, proteolysis, alternative splicing and chromatin remodeling or miscellaneous represent certain regions of the model. Red and blue lightning bolts represent the reception of their respective light wavelengths for each gene symbol. Lines in the model represent known interactions between genes; red arrows are positive interactions, black lines are negative interactions, and blue lines indicate direct physical interactions but the direction, positive or negative, is unknown. No lines indicate that there are no known interactions at this time

these subnetworks, reflecting the complex interplay of positive and negative feedback loops in the circadian clock. Other genes were connected in this clock model through gene module associations, but with no known physical interactions. These genes are new targets for future research on the nature of their interactions within the clock.

\section{Discussion}

The mature berry skin transcriptome was highly dynamic This study investigated grape berry skins sampled at four concentrations of total soluble sugars: $20,22,24$ and 26 ${ }^{\circ}$ Brix. All of these grapes were grown in the same vineyard and thus exposed to nearly identical environmental conditions. We sampled individual berries from a cluster over a narrow developmental range of increasing sugar in an attempt to reduce variability of our samples. Most of the transcriptional changes could be examined with co-expression subnetworks and could be associated with highly enriched GO categories. By comparing both red and white grape cultivars in the same vineyard we could identify modules common to all cultivars that were active in the late ripening stages. In the following sections we discuss some of the processes that appear to be involved in the berry skin gene subnetworks during the late stages of ripening.

\section{Accumulation of sugar and gene expression}

Sugar levels appear to play a role in the transcriptional profiles of the berry skin. PCA and the hclust function utilized in the WGCNA R package showed clear separation by sugar level and a uniformity between samples. These observations were similar to other studies where developmental stages were separated by ${ }^{\circ}$ Brix [57-60]. Grape berries on a cluster can ripen asynchronously with a range of ${ }^{\circ}$ Brix levels [61]. The lack of uniformity in sugar concentration can range from 5 to $7{ }^{\circ}$ Brix within a grape cluster [62]. Indeed, we observed varying ${ }^{\circ}$ Brix within a cluster while separating individual berries from our clusters in this study, which followed a normal distribution within a cluster. Ripening related asynchronicity within a cluster has been shown to synchronize in some situations at maturity [60], but this process was not complete in our berries of seven different varieties, where we observed differences in berries on a single cluster of approximately $4{ }^{\circ}$ Brix (sometimes a difference of $8^{\circ}$ Brix was observed).

Gene expression in grape berries is affected by the sugar concentration [16]. Grape berry sugar concentrations increase substantially after veraison, when soluble sugars are actively transported via the phloem while vines are photosynthetically active $[7,63,64]$. Sugars influence fruit development and gene expression in other 
plant species [11-13]. In grapes, sugar can affect cell growth and induce the transcription of some genes in berries [16]. Sugar increases the expression of a glucose-6phosphate transporter facilitating sucrose transport for starch conversion in plastids [65] and acts as a sensor for sugar signaling [10]. A putative glucose-6phosphate transporter (VIT_19s0177g00300) had increasing transcript abundance with ${ }^{\circ}$ Brix (Additional file 3).

Some bZIP TFs also contain a sucrose-controlled upstream open-reading frame that exhibits repressed expression under increasing molarities of sugar $[66,67]$. The promoter sequence of a dihydroflavonol reductase gene (VIT_18s0001g12800) contains a G-box binding domain, MYB and sucrose box domains that can be induced by sucrose, glucose and fructose, constituents of a ripening berry [68]. Sugar regulates other genes involved in sugar transport and anthocyanin biosynthesis in grape berries [16].

Thus we confirmed our hypothesis that the transcript abundance of genes would vary with sugar level. This argument is supported by the substantial separation of samples by sugar level in the principal component 2 (Fig. 1), which was only slightly lower (21.4\%) than principal component 1 (21.8\%), which separated by genotype. In addition, there was an enrichment of several GO categories for responses to sugar (Additional files 5 and 6). While harvesting grapes at different times may have contributed to some variability in the samples, separating samples based on their sugar level captured a large part of the variability. It is likely that some changes in gene expression in these berry skins were influenced by the sugar concentration.

\section{Autophagy and the decrease of photosynthesis transcripts}

Many ripening related processes were observed in our data. There was an increase in specific hub genes involved in autophagy. The most highly connected hub for autophagy was the ATG18g transcript in the turquoise module (hub \#20; Additional file 11). Autophagy is a degradative process that involves the formation of autophagosomes [54] and intracellular vesicle transport [55]. ATG18g, APG9 (ATG9) and ATG2, all hubs in the turquoise module, are part of the ATG9 cycling system, which participates in the formation of autophagosomes [54]. There were also a number of proteolysis genes in the top 100 hubs of the turquoise module (Additional file 13). Other senescent-like processes have been observed during grape ripening such as chloroplast disintegration [23, 24] and cell death [22].

Ripening also included the continued decrease in transcript abundance of most photosynthetic transcripts that were highly enriched in the darkseagreen 3 module. This is consistent with the deactivation or degradation of chloroplasts; interestingly, the darkseagreen3 module was negatively correlated with the turquoise module indicating that there may be an interaction between the two subnetworks.

Most photosynthesis-related transcripts were decreased in late ripening berries. The ripening berry is a sink organ for photosynthate, losing its photosynthetic capacity with time and changing color as chloroplasts are degraded or converted to other plastids with changing carotenoid production $[46,69]$. In tomato, chloroplasts are converted to chromoplasts, which are the source of the red pigments. In grapes, it appears from two studies that the chloroplasts remain chloroplasts, but that the chloroplasts structure begins to become disorganized with plastoglobules forming and enlarging [23, 24]. These plastoglobules may be a source for lipids used in volatile production during the late stages of fruit ripening [24]. Hardie et al. [24] associated changes in chloroplast structure with the formation of lipid bodies and monoterpenes, which contribute important volatile aromas in maturing grapes. High sugar concentrations can induce senescence [9], repress transcription of the plastome, reduce chloroplast numbers and alter chloroplast morphology [10]. Perhaps the high sugars in the berries have the same effect?

Other ripening processes included cell wall and lipid metabolism. Some cell wall softening genes like a polygalacturonase (VIT_16s0050g01110) and a xyloglucan endotransglycosylase/hydrolase (VIT_02s0012g02220) were positively correlated with ${ }^{\circ} \mathrm{Brix}$ levels in the mature berry skins, whereas the transcript abundance of other cell wall proteins were decreased, such as a group of expansins (Additional file 3). Genes involved in fatty acid and lipid oxidation were also highly enriched in the turquoise module (Additional file 11); the transcript abundance of these genes increased with increasing sugar level.

\section{Epigenetic regulation}

The turquoise module was highly enriched with genes involved with epigenetic regulation. DNA methylation plays an indispensable role in regulating endogenous gene transcription [70]. In general, methylation of genes inhibits gene expression or methylation of RNA affects alternative splicing. Methylation of histones can be permissive for gene expression (e.g. H3K4me3, H3K36me/ me2/me3, and H3K9me3). Acetylation and monoubiquination of histones are also generally associated positively with gene activity. In contrast, histone $\mathrm{H} 2 \mathrm{~A}$ monoubiquitination [71] and H3K27 trimethylation [72] potently repress transcription by the action of the polycomb repressive complexes, PRC2 and PRC1.

Regulation of fruit ripening is linked to DNA methylation. For example, gradual decreases in methylation of the promoter region of the RIN MADS-box TF in tomato [17] allows ripening to proceed. Differentially 
expressed methyltransferases, like $C M T, D R M$ and $M E T$, are transcriptionally active during fruit development in pear [73] and legume [74]. These findings indicate a possible role for normal fruit ripening through the regulation of DNA methylation, particularly in this class of genes highly conserved in eukaryotic species [75].

Methyl-CpG-Binding Domain 9 (MBD9) in Arabidopsis can modulate DNA methylation and histone acetylation to regulate both flowering time and shoot branching by specifically binding methylated $\mathrm{CpG}$ dinucleotides [76-78]. Atmbd9 mutants flower earlier and show abnormal axillary bud outgrowth [77], displaying significantly methylated promoter and intronic regions of the FLC gene [78]. A common increase in transcript abundance of VviMBD9 in all grape cultivars was observed and raises the possibility for methylation of DNA and histones (which presumably causes a reduction in transcription).

Likewise, DDM1 and PIE1 transcript abundance increased in berries with increasing ${ }^{\circ}$ Brix level in the late ripening stages, peaking at $26^{\circ}$ Brix. DDM1 proteins have been observed co-localizing with $M B D$ proteins forming protein complexes [76]. DDM1 in Arabidopsis $[79,80]$ and rice [81] is necessary for genomic DNA methylation and chromatin remodeling through preferential methylation of histone $\mathrm{H} 3$ lysine 9 (H3-K9) instead of transposable elements.

Genome-wide reduction of DNA methylation results in severe developmental and morphological defects in $d d m 1$ mutants [82]. In Arabidopsis, PIE1 forms part of the Swr1-like complex which deposits a histone variant, H2A.Z, onto chromatin around both the transcriptional start and stop sites on genes responsible for flowering repression (FLC, MAF4 (MADS Affecting Flowering 4) and MAF5 (MADS Affecting Flowering 5)) enabling their competence for activation by other factors [83]. Our results support the hypothesis for a role of epigenetic regulation during the late stages of berry development.

\section{Light and the core circadian clock}

Not all DEGs in the berry appear to be related to the sampled ${ }^{\circ}$ Brix levels, some genes appear to be influenced by the genotype (Fig. 1). Light may also be having an effect on gene expression. There are at least two publications that provide evidence for a role of light and the circadian clock in grapevine.

In the first, over a thousand genes in two Vitis vinifera cultivars were recently observed expressing distinctive circadian rhythms throughout the light-dark cycle [84]. For example, VviLHY and VviTOC1 did not oscillate, whereas VviRVE1 and VviELF3 of the core clock genes did display a circadian rhythm in grape [84]. The authors attribute the differences in clock gene expression to grape maintaining a simplified clock in ripening fruit.
Furthermore, secondary processes seemed more responsive to circadian oscillation in late ripening stages than primary metabolism, such as the phenolic pathway enzymes: stilbene synthases and phenylalanine ammonia lyase $[84,85]$.

In the second publication, a genome-wide analysis of the cis-regulatory elements (CREs) of grapevine protein-coding gene promoters was performed [86]. Highly enriched modules or gene networks were identified. There are over 4000 promoters with a circadian clock-associated CRE. The genes with this CRE in their promoters are highly enriched in functional categories associated with abiotic stress, hormone, flavonoid, and isoprenoid metabolism. There are a large number of chalcone synthase, stilbene synthase, and terpene synthase genes in this group. The authors suggested that grapevine might have an "expanded clock regulatory network". In addition, they linked light- and chloroplast-related genes to the subnetwork of the circadian clock gene, $V v i H Y H$.

The circadian clock is regulated by light and may have affected gene expression in grape berry skins during the late stages of berry ripening. Day length decreased with advanced berry ripening and the progression of autumn.

Numerous genes in the core circadian clock were highly connected in gene subnetworks in the late ripening stage. Many of the core clock genes displayed similar patterns of expression for all genotypes (Fig. 8). As the day length shortened, it appears that the day phase of the clock accelerated or shortened, allowing a greater involvement of the evening complex and its cyclic repression of the morning genes.

The circadian clock is regulated by a number of negative feedback loops and positive regulators [87, 88]. Its regulation involves a complicated interaction of transcription [89], RNA splicing [90, 91], proteolysis [92] and histone modifications [53, 93]. Light regulates histone modifications, gene expression and higher order chromosome organization [53]. Genes from all of these processes are present in our circadian clock model (Fig. 8).

The circadian clock regulates gene expression throughout the day and night. It regulates the rhythmic variation in photosynthesis, starch accumulation, and starch turnover [94]. A well-supported hypothesis is that the circadian clock regulates starch metabolism in the leaf to maintain sugar metabolism throughout the night. The circadian clock in turn is sensitive to photosynthesis and soluble sugar accumulation [95]. Sugars accelerate the day phase of the circadian clock.

Do sugars accelerate the day phase in berries? The role of the circadian clock is well studied in leaves, roots, apical meristems, buds and flowers, but there are few papers on the role of the circadian clock in fruit development.

Light signals set the circadian clock (Fig. 8) as the day length shortens in autumn. The circadian clock likely 
received day length signals through far red (e.g. FAR1related proteins) and blue light sensors (e.g. ZTL) that trigger changes in gene expression involving RNA processing, proteolysis, chromosome modification and organization, and may lead to major changes in berry metabolism (e.g. suppression of gene expression of chloroplast genes).

Yet berries develop normally in grapevines grown under constant environmental conditions in the greenhouse [96, 97] or growth chamber [11]. Elevated $\mathrm{CO}_{2}$ concentrations substantially accelerate photosynthesis and berry development [97], indicating that sugar accumulation may affect rates of berry development. Can changes in day length or the circadian clock alter the rate of berry development and fruit composition as well? To what extent the circadian clock functions in berry development is unclear, but the network analyses results in this study indicate that it was highly connected to major developmental processes and gene modules related to epigenetic regulation and chromosome organization. The interactions of sugar with the circadian clock may be even more complex. The roles and interactions of the circadian clock with sugar accumulation and grape berry development warrants further investigation.

\section{Conclusions}

Gene expression of berry skins in the late stages of ripening was associated with sugar accumulation and genotype. A common set of genes for all seven cultivars was identified in the late ripening stages of berry development. Transcriptional regulation of fruit ripening involved many transcription factors and other regulators of hormone levels. In addition, the transcript abundance of genes related to DNA methylation indicated that epigenetic programming might be involved in the regulation of transcription during berry ripening at maturity, suppressing or silencing many genes. Gene co-expression analysis was used by two different approaches to elucidate complex transcriptional networks. Two of the most highly connected gene subnetworks consisted of hundreds of genes: one subnetwork involved RNA processing, chromosome organization, epigenetic regulation, proteolysis and autophagy and the other subnetwork, negatively correlated with the first, involved photosynthesis. Thus, there seems to be an interaction between these two subnetworks that links nucleotide metabolism and autophagy with berry skin ripening processes including the large decrease in photosynthetic transcripts. A circadian clock signature for key clock components was also observed to participate in these subnetworks and warrants further study to better understand the role light plays in these subnetworks and the late stages of berry ripening.
The identification of a core set of genes common to all seven cultivars, both red and white wine producing, allowed the identification of key processes in the development of late ripening berry skins, including autophagy, catabolism, nucleotide metabolism, photosynthesis, cell wall metabolism, gene expression and chromosome organization. Some of these key processes were associated with specific modules or gene subnetworks and the key hub genes in these subnetworks were ranked for future targeted testing. These transcriptomic results support the hypothesis that senescent-like processes dominate the late stages of berry ripening as the seed matures and prepares for dispersal.

\section{Methods}

\section{Plant materials}

Vitis vinifera L. cultivars Cabernet Franc, Cabernet Sauvignon, Merlot, Pinot Noir, Chardonnay, Sauvignon Blanc and Semillon were grown at the University of Nevada, Reno's Nevada Agricultural Experiment Station Valley Road Experimental Vineyard. Research approval was obtained by Grant R. Cramer from the Nevada Agricultural Experiment Station and the University of Nevada, Reno. All grape cultivars were originally obtained as certified material from Inland Desert Nursery, Benton, City, Washington, USA. The cultivars were surveyed in September and October 2012, depending upon the berry maturity of each cultivar. Maturity was assessed using a digital refractometer (HI 96811, Hanna Instruments, Woonsocket, RI, USA) to measure soluble solids ( ${ }^{\circ}$ Brix) that are mostly made up of sugars. Berry clusters were collected between $11.00 \mathrm{~h}$ and $13.00 \mathrm{~h}$ (near solar noon) in an attempt to minimize temporal transcriptional response variations caused by the circadian clock. At harvest, individual berry ${ }^{\circ}$ Brix levels were determined with a digital refractometer. Separated berry skins were placed into $50 \mathrm{~mL}$ centrifuge tubes in liquid nitrogen according to sugar level $\left(1 \pm 0.5{ }^{\circ}\right.$ Brix increments; 19 to $27^{\circ}$ Brix). Berry to berry variation was considerable on an individual cluster; in some cases berries varied as much as $8^{\circ} \mathrm{Brix}$ on a single cluster. In this way berries were collected over many days from multiple clusters from multiple vines from 3 different individually irrigated blocks in the vineyard. Each block was considered an experimental replicate.

\section{RNA extraction}

Three experimental replicates from each cultivar at 20, 22, 24 and $26{ }^{\circ}$ Brix were used for sequencing. Total RNA was extracted from approximately $250 \mathrm{mg}$ of finely ground skin tissue using a modified CTAB extraction protocol followed by an additional on-column DNase digestion using a Qiagen RNeasy Mini Kit (Qiagen, Valencia, CA, USA) as in [33]. RNA quality and quantity 
were assessed with a Nanodrop ND-1000 spectrophotometer (ThermoFisher Scientific, Waltham, MA, USA) and an Agilent 2100 Bioanalyzer (Agilent Technologies, Santa Clara, CA, USA).

\section{RNA-seq library preparation and sequencing}

Eighty-four 50 bp single-end, barcoded libraries were constructed and sequenced by the Neuroscience Genomics Core at the University of California, Los Angeles (Los Angeles, CA, USA) using Illumina TruSeq RNA library prep kits (Illumina Inc., San Diego, CA, USA) according to the manufacturer's instructions. The barcoded libraries were pooled and multiplexed, and were sequenced using Illumina TruSeq chemistry (version 3.0) on a HiSeq2000 sequencer (Illumina Inc., San Diego, CA, USA).

\section{Gene expression and statistical analysis}

The single-end sequence fragments (reads) generated by Illumina sequencing were base-called, demultiplexed, and then quality filtered with the NGS QC Toolkit [98]. TopHat2 (version 2.0.10) was used to align reads to the V1 version of the PN40024 Vitis vinifera reference genome annotation obtained at EnsemblPlants [99]. Filtered reads were aligned with the "-b2-very-sensitive" option for Tophat 2 and "-transcriptome-index" option with a corresponding EnsemblPlants-sourced index, as in [33, 100]. Remaining parameters were kept at default. Approximately 93\% of reads were mapped (Additional file 1). Samtools [101] and HTSeq [102] were used to generate feature counts from the BAM alignment files produced by Tophat2. HTSeq was run using the "union" mode, with the "-i gene_id -t exon -s no" options. Counts of genes were filtered prior to expression analysis, using the following independent criteria: transcripts with zero counts in all samples were excluded; further filtering was performed to remove transcripts with $<15$ counts in $75 \%$ of samples $(63$ of 84) prior to expression comparison (Additional file 2). Correction scaling factors were computed from the filtered and untransformed library counts using the trimmed mean of M-values method between each pair of sample libraries, as implemented in edgeR (3.14.0) [103]. This produced an effective library size for model-based differential expression analysis in edgeR. A design model that defined each ${ }^{\circ}$ Brix level combination as an element of a group $(\sim 0+$ Group $)$ was used to test for differential expression using simple contrasts between subgroups of interest within each cultivar (e.g. 22-20, 24-20, 24-22, 26-20, 26-22, 26-24). Statistically significant transcript abundance changes were found below the adjusted $p$-value of 0.05 , according to the Benjamini and Hochberg procedure to control the false discovery rate (FDR) [104]. An intersection of statistically significant transcripts at different ${ }^{\circ}$ Brix levels was made to derive a common set of berry skin transcripts shared in the seven grapevine cultivars. RNA-seq statistics are presented in Table 1.

\section{Gene co-expression analysis}

Gene expression was also evaluated with the WGCNA R package version 1.51 [27] to identify modules of correlated genes and investigate intramodular hub genes. A variance-stabilizing transformation of the filtered counts of genes was performed using DESeq2 version 1.12.3 [105] before WGCNA, as suggested by WGCNA package authors. To meet the scale-free topology criteria for optimal clustering, a power of 11 was selected as a soft threshold value to transform the adjacency matrix (see Additional file 10); a biweight midcorrelation was used as an alternative to Pearson's correlation. The following settings for the adjacency function (datExpr, power $=11$, type = "signed hybrid", corFnc = "bicor", corOptions = "use $=$ 'p', maxPOutliers $=0.1$ ") and for the cuttreeDynamic function (dendro $=$ geneTree, distM $=$ dissTOM, deepSplit $=2$, pamRespectsDendro $=$ FALSE, minClusterSize $=30$ ); these functions have been shown to be the best approach for biologically meaningful results [26].

The petal $\mathrm{R}$ package [29] was independently utilized to construct a true small-world and scale-free complex gene co-expression network model from which gene modules with higher intra-correlation and density were identified. Before creating a network model, the distribution of the entire dataset of 16,606 filtered and qualitycontrolled transcripts was investigated for normality to

Table 1 RNA-sequencing, read mapping and feature count statistics of the experiment

\begin{tabular}{|c|c|}
\hline Next-generation sequencing platform & Illumina Hiseq2000 \\
\hline Cultivars investigated & 7 \\
\hline Library type & Single-end \\
\hline Number of libraries & 84 \\
\hline Read length (bp) & 50 \\
\hline Total number of reads & $2,901,803,214$ \\
\hline Average total reads & $34,545,276.36$ \\
\hline Total number of bases $(\mathrm{Gb})^{\mathrm{a}}$ & 145.09 \\
\hline Total number of $\mathrm{HQ}^{\mathrm{b}}$ filtered reads & $2,877,839,522$ \\
\hline Average $\mathrm{HQ}$ reads & $34,259,994.31$ \\
\hline Total number of HQ bases in HQ reads (Gb) & 143.89 \\
\hline Percentage of $\mathrm{HQ}$ filtered reads & $99.18 \%$ \\
\hline alignment_not_unique ${ }^{c}$ & $3,410,081.85$ \\
\hline ambiguous $^{c}$ & $219,495.24$ \\
\hline no_feature ${ }^{c}$ & $2,692,823.24$ \\
\hline not_aligned ${ }^{c}$ & 0.00 \\
\hline too_low_aQual ${ }^{\mathrm{C}}$ & 0.00 \\
\hline
\end{tabular}

${ }^{a}$ Giga base pair $(1,000,000,000 \mathrm{bp})$

${ }^{\mathrm{b}}$ High quality (Phred score $=20$ )

'HTSeq 
ensure statistically appropriate association measures to define genes as similar. The graph generated by graphHistQQFromFile clearly showed a non-normal distribution (Additional file 16); thus, the association measure, Spearman Correlation Coefficient, was left at default and createSWSFnetFromFile was used to create the network model with no other parameter settings. The model's hub genes were identified by calculating the connectivity degree (number of neighbors) of each node (gene, transcript) using the function: getVertexStats.

\section{Gene annotation and functional enrichment of GO \\ categories}

Genes were annotated from three sources. Arabidopsis orthologs were identified using The Arabidopsis Information Resources (TAIR) annotation in Gramene [106]. Additional annotations were added from Grimplet et al. [107] and the corresponding author. A GO file was created using the EnsemblPlants BioMart [108] for Vitis vinifera. Functional category enrichment of biological processes was determined with the BinGO plugin application (version 3.0.2) in Cytoscape (version 3.2.1) [109]. Gene ontology membership classifies function hierarchically from broad to specific. Multiple testing correction adjusted $p$-values were determined using the Benjamini \& Hochberg false discovery rate (FDR) at a 0.05 threshold.

\section{Additional files}

Additional file 1: Read counts uniquely mapped to the PN40024 grape genome (XLSX $12905 \mathrm{~kb}$ ).

Additional file 2: $\log _{2}$ counts per million of filtered and normalized read counts (XLSX $11815 \mathrm{~kb}$ ).

Additional file 3: Differential expression results from edgeR of the significantly affected transcripts in common from berry skins of seven grapevine cultivars at different ${ }^{\circ}$ Brix levels. V1 ID refers to the transcript identification of the gene loci of the V1 version of the PN40024 Vitis vinifera reference genome. Cultivar abbreviations are Cabernet Sauvignon (CS), Merlot (ME), Pinot Noir (PN), Chardonnay (CD) and Semillon (SM). P-values and adjusted $p$-values (PAdj) were calculated for each of the comparisons between the different ${ }^{\circ}$ Brix levels for each cultivar (XLSX 2391 kb).

Additional file 4: Annotation of the differentially expressed genes (DEGs) and count data associated with each gene. V1 ID is the same as in Additional file 3. AtID refers to Arabidopsis thaliana gene loci. Headings on the column refer to the cultivar abbreviation followed by the number of the replicate and the number of the ${ }^{\circ} B r i x$ level. The final columns compute the differences between ${ }^{\circ} B r i x$ levels for each cultivar and average them for all cultivars. Color highlights represent decreasing (green) or increasing (red) differences for the gene for the 26 to $20{ }^{\circ}$ Brix comparison (26-20) (XLSX 3136 kb).

Additional file 5: Statistical results from gene set enrichment analysis of the DEGs using BinGO (XLSX $271 \mathrm{~kb}$ ).

Additional file 6: Image of the network construction of the gene ontology categories by BinGO. Yellow colors represent significant enrichment and size of the circle represents the number of genes in each set (PDF $214 \mathrm{~kb}$ ).

Additional file 7: BinGO analysis of the top 500 DEGs with a positive difference between 26 and $20^{\circ}$ Brix (26-20) (XLSX $\left.73 \mathrm{~kb}\right)$.
Additional file 8: BinGO analysis of the top $500 \mathrm{DEGs}$ with a negative difference between 26 and $20^{\circ}$ Brix (26-20) (XLSX $88 \mathrm{~kb}$ ).

Additional file 9: A gene cluster dendrogram with gene modules (colored blocks) for the cuttreeDynamic and merged modules functions in WGCNA (PDF $11 \mathrm{~kb}$ ).

Additional file 10: Scale independence and connectivity plots for the determination of the power function of a scale-free topology using the soft thresholding function in WGCNA (PDF $5 \mathrm{~kb}$ ).

Additional file 11: Module membership of all filtered transcripts as defined by WGCNA. Values are the kME (module eigengene connectivity). Circadian rhythm and clock genes are highlighted in yellow (XLSX $17289 \mathrm{~kb})$.

Additional file 12: Summary details of all 64 gene modules. Gene module rows are highlighted with different colors to reflect transcript abundance differences between red and white grapes for each module (red highlight for red grapes, green highlight for white grapes and yellow highlight for no distinction between red and white grapes) (XLSX $99 \mathrm{~kb}$ ).

Additional file 13: Annotations of the top 100 hub (kME) genes in the turquoise module. Highlight colors represent different $\mathrm{GO}$ categories assigned to each gene (XLSX $111 \mathrm{~kb}$ ).

Additional file 14: Network statistics for the petal analysis on the filtered set of 15,092 genes. V1 ID is as in Additional file 3. The table includes all genes of the network model based on a Spearman correlation threshold of 0.703. Each gene's cluster coefficient (localCC) and connectivity degree ( $k$ ) are included (XLSX $423 \mathrm{~kb}$ ).

Additional file 15: The set of 216 genes in the hub module constructed from the top 5\% of the most connected genes in the petal-constructed gene network. Column headings are the same as in previous additional files (XLSX $76 \mathrm{~kb}$ ).

Additional file 16: Top) Histogram (blue) of the 16,606 transcripts and line (line) of expected normal distribution considering the mean and standard deviation of the given data. Bottom) Quantile-quantile $(\mathrm{Q}-\mathrm{Q})$ plot of given data compared to the expected hypothetical normal distribution. Histogram and Q-Q plots demonstrate that the distribution of the given data is not normal as the histogram lies outside the normal curve and the points on the QQ-plot are not scattered alone the red line (PDF $580 \mathrm{~kb}$ ).

\section{Abbreviations}

2 ATG11: AuTophaGy-related 11; ABA: Abscisic acid; APG9: AutoPhaGy 9; ARC5: Accumulation and Replication of Chloroplast 5; ARF3: Auxin Response Factor 3; ASR: Abscisic acid Stress Ripening; ATG18g: Homolog of yeast AuTophaGy 18 g; ATG2: AuTophaGy; BARD: United States - Israel Binational Agricultural Research and Development Fund; Bp: Base pair; CCA1: Circadian Clock Associated 1; CIP4: COP1 Interacting Protein 4; COL4: COnstans-Like 4; COP1: COnstitutive Photomorphogenic 1 CREs: cis-regulatory elements; CRY3: CRYptochrome 3; CURT1: CURvature Thylakoid 1; DDM1: Decrease in DNA Methylation 1; DEG(s): Differentially Expressed Gene(s); EFS: Early Flowering in Short days; ELF: EarLy Flowering; EMF1: Embryonic Flower 1; FAR1: Far-Red impaired Response 1; FDR: False Discovery Rate;

FLC: Flowering Locus C; FRS5: FAR1-Related Sequence 5; FT: Flowering locus T; GO: Gene Ontology; GPT2: Glucose Phosphate Transporter 2;

HDA6: Histone DeAcetylase 6; HKT1: High-affinity K (potassium) Transporter 1; HMG: High Mobility Group; HSL1: HAESA-like 1; HUB: Histone Ubiquitin ligase; HXK1: HeXose Kinase; HY5: Elongated HYpocotyl 5; kME: Module Eigengene connectivity; LHY: Late elongated HYpocotyl; LNK2: LightiNducible and clock-regulated 2; MAF4: MADS Affecting Flowering 4; MAF5: MADS Affecting Flowering 5; MBD9: Methyl-CPG-Binding Domain 9; MCM: MiniChromosome Maintenance; ME: Module Eigengene; MORC4: MicrORChidia 4; NCBI: National Center for Biotechnology Information; NPH3: Non-Phototropic Hypocotyly 3; OPR3: OxoPhytodienoate Reductase 3; PCA: Principal Component Analysis; PcG: Polycomb group; PCL1: PhytoCLock 1; PHYB: PHYtochrome B; PHYE: PHYtochrome E; PIE1: Photoperiod Independent Early Flowering 1; PPR: PentatricoPeptide Repeat; PRR9: Psuedo-Response Regulator 9; PTAC16: Plastid Transcriptionally Active 16; RCC1: Regulator of Chromosome Condensation; RVE1: ReVeillE 1; SKIP: SNW/SKI-Interacting Protein; SNF: Sucrose Non-Fermenting; SnRK1.1: SNF1 Related protein Kinase; SPA3: Suppressor of Phytochrome A-related 3; TAIR: The Arabidopsis Information Resource; TCP7: Transcription factor TCP domain protein 7; TF(s): Transcription Factor(s); TIC: Time for 
Coffee; TOR: Target Of Rapamycin; TPL: ToPLess; Vvi: Vitis vinifera; WGCNA: Weighted Gene Co-expression Network Analysis; XCT: XAP5 Circadian Timekeeper; ZTL: ZeiTLupe

\section{Acknowledgements}

We acknowledge and thank the many UNR biochemistry senior thesis undergraduate students that assisted in berry separation by ${ }^{\circ}$ Brix level.

\section{Funding}

This research was supported by Research Grant Award No. IS-4325-10 from BARD, The United States - Israel Binational Agricultural Research and Development Fund to GRC and AF. Bioinformatic facilities, RLT and KAS were supported by a grant from the National Institute of General Medical Sciences (GM103440).

\section{Availability of data and materials}

RNA-seq data were deposited with the Sequence Read Archive database at the National Center for Biotechnology Information (NCBI) with BioProject identifier PRJNA260535 [110].

\section{Authors' contributions}

RG designed and conducted the experiments, analyzed the data and wrote the body of the paper; JP conducted the petal analysis; RLT converted and filtered the RNA-seq raw data; KAS advised RG on statistical analysis; DT helped on the WGCNA analysis; AF assisted on experimental design, analysis of the data and acquiring funds for the project; GRC helped acquire the funding; he supervised all aspects of the project including writing and editing the final version of the manuscript. All authors reviewed, edited and approved the final version of the manuscript.

\section{Competing interests}

Drs. Aaron Fait and Grant R. Cramer are editorial board members of BMC Plant Biology.

\section{Consent for publication}

Not applicable.

\section{Ethics approval and consent to participate}

Not applicable.

\section{Publisher's Note}

Springer Nature remains neutral with regard to jurisdictional claims in published maps and institutional affiliations.

\section{Author details}

'Department of Biochemistry and Molecular Biology, University of Nevada, Reno, NV 89557, USA. ${ }^{2}$ Nevada INBRE Bioinformatics Core, University of Nevada, Reno, NV 89557, USA. ${ }^{3}$ Telekom Innovation, Laboratories and Cyber Security Research Center, Department of Information, Systems Engineering, Ben Gurion University, Beer Sheva, Israel. ${ }^{4}$ Ben-Gurion University of the Negev, Jacob Blaustein Institutes for Desert Research, 84990 Midreshet Ben-Gurion, Israel.

Received: 20 September 2016 Accepted: 22 May 2017

\section{Published online: 30 May 2017}

\section{References}

1. Seymour GB, Ostergaard L, Chapman NH, Knapp S, Martin C. Fruit development and ripening. Annu Rev Plant Biol. 2013;64:219-41.

2. Kuhn N, Guan L, Dai ZW, Wu BH, Lauvergeat V, Gomes E, et al. Berry ripening: recently heard through the grapevine. J Exp Bot. 2014;65(16): 4543-59.

3. Yang J, Xiao YY. Grape phytochemicals and associated health benefits. Crit Rev Food Sci Nutr. 2013;53(11):1202-25.

4. Jimenez-Garcia SN, Guevara-Gonzalez RG, Miranda-Lopez R, Feregrino-Perez AA, Torres-Pacheco I, Vazquez-Cruz MA. Functional properties and quality characteristics of bioactive compounds in berries: biochemistry, biotechnology, and genomics. Food Res Int. 2013:54(1):1195-207.

5. Zenoni S, Fasoli M, Guzzo F, Dal Santo S, Amato A, Anesi A, et al. Disclosing the molecular basis of the postharvest life of berry in different grapevine genotypes. Plant Physiol. 2016;172(3):1821-43.
6. Sheen J. Master regulators in plant glucose signaling networks. J Plant Biol. 2014;57(2):67-79.

7. Conde C, Silva P, Fontes N, Dias A, Tavares R, Sousa M, et al. Biochemical changes throughout grape berry development and fruit and wine quality. FoodReview. 2007;1:1-22.

8. Lastdrager J, Hanson J, Smeekens S. Sugar signals and the control of plant growth and development. J Exp Bot. 2014;65(3):799-807.

9. Pourtau N, Jennings R, Pelzer E, Pallas J, Wingler A. Effect of sugar-induced senescence on gene expression and implications for the regulation of senescence in Arabidopsis. Planta. 2006;224(3):556-68.

10. Van Dingenen J, De Milde L, Vermeersch M, Maleux K, De Rycke R, De Bruyne $\mathrm{M}$, et al. Chloroplasts are central players in sugar-induced leaf growth. Plant Physiol. 2016;171(1):590-605.

11. Dai ZW, Meddar M, Renaud C, Merlin I, Hilbert G, Delrot S, et al. Long-term in vitro culture of grape berries and its application to assess the effects of sugar supply on anthocyanin accumulation. J Exp Bot. 2014;65(16):4665-77.

12. Jia H, Jiu S, Zhang C, Wang C, Tariq P, Liu Z, et al. Abscisic acid and sucrose regulate tomato and strawberry fruit ripening through the abscisic acidstress-ripening transcription factor. Plant Biotechnol J. 2016;14(10):2045-65.

13. Kong L, Guo H, Sun M. Signal transduction during wheat grain development. Planta. 2015;241(4):789-801.

14. Cakir B, Agasse A, Gaillard C, Saumonneau A, Delrot S, Atanassova R. A grape ASR protein involved in sugar and abscisic acid signaling. Plant Cell. 2003;15(9):2165-80.

15. Lecourieux $F$, Lecourieux $D$, Vignault $C$, Delrot $S$. A sugar inducible protein kinase, VVSK1, regulates hexose transport and sugar accumulation in grapevine cells. Plant Physiol. 2010;52:1096-106.

16. Lecourieux F, Kappel C, Lecourieux D, Serrano A, Torres E, Arce-Johnson P, et al. An update on sugar transport and signalling in grapevine. J Exp Bot. 2014:65(3):821-32.

17. Zhong S, Fei Z, Chen YR, Zheng Y, Huang M, Vrebalov J, et al. Single-base resolution methylomes of tomato fruit development reveal epigenome modifications associated with ripening. Nat Biotechnol. 2013;31(2):154-9.

18. Almada R, Cabrera N, Casaretto JA, Pena-Cortes H, Ruiz-Lara S, Gonzalez Villanueva E. Epigenetic repressor-like genes are differentially regulated during grapevine (Vitis vinifera L.) development. Plant Cell Rep. 2011; 30(10):1959-68

19. Aquea F, Vega A, Timmermann T, Poupin MJ, Arce-Johnson P. Genomewide analysis of the SET DOMAIN GROUP family in grapevine. Plant Cell Rep. 2011;30(6):1087-97.

20. Cramer GR, Ghan R, Schlauch KA, Tillett RL, Heymann H, Ferrarini A, et al. Transcriptomic analysis of the late stages of grapevine (Vitis vinifera $\mathrm{cV}$. Cabernet Sauvignon) berry ripening reveals significant induction of ethylene signaling and flavor pathways in the skin. BMC Plant Biol. 2014;14(1):370.

21. Zamboni A, Minoia L, Ferrarini A, Tornielli GB, Zago E, Delledonne M, et al. Molecular analysis of post-harvest withering in grape by AFLP transcriptional profiling. J Exp Bot. 2008:59(15):4145-59.

22. Tilbrook J, Tyerman SD. Cell death in grape berries: varietal differences linked to xylem pressure and berny weight loss. Funct Plant Biol. 2008:35(3):173-84.

23. Diakou P, Carde JP. In situ fixation of grape berries. Protoplasma. 2001; 218(3-4):225-35

24. Hardie WJ, Aggenbach SJ, Jaudzems VG. The plastids of the grape pericarp and their significance in isoprenoid synthesis. Aust J Grape Wine Res. 1996; 2(3):144-54

25. Heymann H, LiCalzi M, Cionversano MR, Bauer A, Skogerson K, Matthews M. Effects of extended grape ripening with or without must and wine alcohol manipulations on Cabernet Sauvignon wine sensory characteristics. S Afr J Enol Vitic. 2013;34:86-99.

26. Song L, Langfelder P, Horvath S. Comparison of co-expression measures: mutual information, correlation, and model based indices. BMC Bioinformatics. 2012;13:328.

27. Langfelder P, Horvath S. WGCNA: an R package for weighted correlation network analysis. BMC Bioinformatics. 2008;9:559.

28. Langfelder $\mathrm{P}$, Horvath S. Eigengene networks for studying the relationships between co-expression modules. BMC Syst Biol. 2007;1:54.

29. Petereit J, Smith S, Harris FC Jr, Schlauch KA. Petal: co-expression network modelling in R. BMC Syst Biol. 2016;10(Suppl 2):51.

30. Kobayashi Y, Sadhukhan A, Tazib T, Nakano Y, Kusunoki K, Kamara M, et al. Joint genetic and network analyses identify loci associated with root growth under $\mathrm{NaCl}$ stress in Arabidopsis thaliana. Plant Cell Environ. 2016; 39(4):918-34. 
31. Amrine KC, Blanco-Ulate B, Cantu D. Discovery of core biotic stress responsive genes in Arabidopsis by weighted gene co-expression network analysis. Plos One. 2015;10(3):e0118731.

32. Hopper DW, Ghan R, Schlauch KA, Cramer GR. Transcriptomic network analyses of leaf dehydration responses identify highly connected ABA and ethylene signaling hubs in three grapevine species differing in drought tolerance. BMC Plant Biol. 2016;16(1):118

33. Ghan R, Van Sluyter SC, Hochberg U, Degu A, Hopper DW, Tillet RL, et al. Five omic technologies are concordant in differentiating the biochemical characteristics of the berries of five grapevine (Vitis vinifera L.) cultivars. BMC Genomics. 2015;16(1):946.

34. Naithani S, Raja R, Waddell EN, Elser J, Gouthu S, Deluc LG, et al. VitisCyc: a metabolic pathway knowledgebase for grapevine (Vitis vinifera). Front Plant Sci. 2014:5:644

35. Hu Y, Zhu N, Wang X, Yi Q, Zhu D, Lai Y, et al. Analysis of rice Snf2 family proteins and their potential roles in epigenetic regulation. Plant Physiol Biochem. 2013;70:33-42.

36. Knizewski L, Ginalski K, Jerzmanowski A. Snf2 proteins in plants: gene silencing and beyond. Trends Plant Sci. 2008;13(10):557-65.

37. Sto IM, Orr RJ, Fooyontphanich K, Jin X, Knutsen JM, Fischer U, et al. Conservation of the abscission signaling peptide IDA during angiosperm evolution: withstanding genome duplications and gain and loss of the receptors HAE/HSL2. Front Plant Sci. 2015;6:931.

38. Chhun T, Chong SY, Park BS, Wong EC, Yin JL, Kim M, et al. HSI2 repressor recruits MED13 and HDA6 to down-regulate seed maturation Gene expression directly during Arabidopsis early seedling growth. Plant Cell Physiol. 2016;57(8):1689-706.

39. Tsukagoshi H, Morikami A, Nakamura K. Two B3 domain transcriptional repressors prevent sugar-inducible expression of seed maturation genes in Arabidopsis seedlings. Proc Natl Acad Sci U S A. 2007;104(7):2543-7.

40. Sharma N, Bender Y, Boyle K, Fobert PR. High-level expression of sugar inducible gene2 (HSI2) is a negative regulator of drought stress tolerance in Arabidopsis. BMC Plant Biol. 2013;13:170.

41. Furutani M, Sakamoto $N$, Yoshida S, Kajiwara T, Robert HS, Friml J, et al. Polar-localized NPH3-like proteins regulate polarity and endocytosis of PIN-FORMED auxin efflux carriers. Development. 2011;138(10):2069-78.

42. Schaller F, Biesgen C, Mussig C, Altmann T, Weiler EW. 12-Oxophytodienoate reductase 3 (OPR3) is the isoenzyme involved in jasmonate biosynthesis. Planta. 2000;210(6):979-84.

43. Valverde F. CONSTANS and the evolutionary origin of photoperiodic timing of flowering. J Exp Bot. 2011;62(8):2453-63.

44. Almada R, Cabrera N, Casaretto JA, Ruiz-Lara S, Gonzalez Villanueva E. VvCO and VVCOL1, two CONSTANS homologous genes, are regulated during flower induction and dormancy in grapevine buds. Plant Cell Rep. 2009; 28(8):1193-203.

45. Griffiths S, Dunford RP, Coupland G, Laurie DA. The evolution of CONSTANS-like gene families in barley, rice, and Arabidopsis. Plant Physiol. 2003;131(4):1855-67.

46. Young PR, Lashbrooke JG, Alexandersson E, Jacobson D, Moser C, Velasco R, et al. The genes and enzymes of the carotenoid metabolic pathway in Vitis vinifera L. BMC Genomics. 2012;13:243.

47. Huang CF, Miki D, Tang K, Zhou HR, Zheng Z, Chen W, et al. A pre-mRNAsplicing factor is required for RNA-directed DNA methylation in Arabidopsis. Plos Genet. 2013;9(9):e1003779.

48. Yamamoto YY, Deng X-W, Matsui M. CIP4, a new COP1 target, is a nucleuslocalized positive regulator of Arabidopsis Photomorphogenesis. Plant Cell. 2001;13(2):399-411.

49. Huang H, Alvarez S, Bindbeutel R, Shen Z, Naldrett MJ, Evans BS, et al. Identification of evening complex associated proteins in Arabidopsis by affinity purification and mass spectrometry. Mol Cell Proteomics. 2016;15(1):201-17.

50. Wang L, Kim J, Somers DE. Transcriptional corepressor TOPLESS complexes with pseudoresponse regulator proteins and histone deacetylases to regulate circadian transcription. Proc Natl Acad Sci U S A. 2013;110(2):761-6.

51. Causier B, Ashworth M, Guo W, Davies B. The TOPLESS interactome: a framework for gene repression in Arabidopsis. Plant Physiol. 2012; 158(1):423-38.

52. Li Y, Mukherjee I, Thum KE, Tanurdzic M, Katari MS, Obertello M, et al. The histone methyltransferase SDG8 mediates the epigenetic modification of light and carbon responsive genes in plants. Genome Biol. 2015;16:79.

53. Barneche F, Malapeira J, Mas P. The impact of chromatin dynamics on plant light responses and circadian clock function. J Exp Bot. 2014; 65(11):2895-913.
54. Liu Y, Bassham DC. Autophagy: pathways for self-eating in plant cells. Annu Rev Plant Biol. 2012;63:215-37.

55. Zientara-Rytter K, Sirko A. To deliver or to degrade - an interplay of the ubiquitin-proteasome system, autophagy and vesicular transport in plants. FEBS J. 2016;283(19):3534-55

56. Armbruster U, Labs M, Pribil M, Viola S, Xu W, Scharfenberg M, et al. Arabidopsis CURVATURE THYLAKOID1 proteins modify THYLAKOID architecture by inducing membrane curvature. Plant Cell. 2013;25(7):2661-78.

57. Pilati S, Brazzale D, Guella G, Milli A, Ruberti C, Biasioli F, et al. The onset of grapevine berry ripening is characterized by ROS accumulation and lipoxygenase-mediated membrane peroxidation in the skin. BMC Plant Biol. 2014;14:87.

58. Lijavetzky D, Carbonell-Bejerano P, Grimplet J, Bravo G, Flores P, Fenoll J, et al. Berry flesh and skin ripening features in Vitis vinifera as assessed by transcriptional profiling. Plos One. 2012;7(6):e39547.

59. Dai ZW, Leon C, Feil R, Lunn JE, Delrot S, Gomes E. Metabolic profiling reveals coordinated switches in primary carbohydrate metabolism in grape berry (Vitis vinifera L.), a non-climacteric fleshy fruit. J Exp Bot. 2013;64(5):1345-55.

60. Gouthu S, O'Neil ST, Di Y, Ansarolia M, Megraw M, Deluc LG. A comparative study of ripening among berries of the grape cluster reveals an altered transcriptional programme and enhanced ripening rate in delayed berries. J Exp Bot. 2014;65(20):5889-902.

61. Coombe BG. Research on development and ripening of the grape berry. Am J Enol Vitic. 1992:43:101-10.

62. Pagay V, Cheng L. Variability in berry maturation of concord and Cabernet Franc in a cool climate. Am J Enol Vitic. 2010;61(1):61-7.

63. Keller M, Smith JP, Bondada BR. Ripening grape berries remain hydraulically connected to the shoot. J Exp Bot. 2006;57(11):2577-87.

64. Choat B, Gambetta GA, Shackel KA, Matthews MA. Vascular function in grape berries across development and its relevance to apparent hydraulic isolation. Plant Physiol. 2009;151(3):1677-87.

65. Noronha $\mathrm{H}$, Conde C, Delrot S, Geros H. Identification and functional characterization of grapevine transporters that mediate glucose-6phosphate uptake into plastids. Planta. 2015;242(4):909-20.

66. Wiese A, Elzinga N, Wobbes B, Smeekens S. Sucrose-induced translational repression of plant bZIP-type transcription factors. Biochem Soc Trans. 2005; 33(Pt 1):272-5.

67. Thalor SK, Berberich T, Lee SS, Yang SH, Zhu X, Imai R, et al. Deregulation of sucrose-controlled translation of a bZIP-type transcription factor results in sucrose accumulation in leaves. Plos One. 2012;7(3):e33111.

68. Gollop R, Even S, Colova-Tsolova V, Perl A. Expression of the grape dihydroflavonol reductase gene and analysis of its promoter region. J Exp Bot. 2002;53(373):1397-409.

69. Fanciullino AL, Bidel LP, Urban L. Carotenoid responses to environmental stimuli: integrating redox and carbon controls into a fruit model. Plant Cell Environ. 2014;37(2):273-89.

70. Zilberman D, Gehring M, Tran RK, Ballinger T, Henikoff S. Genome-wide analysis of Arabidopsis thaliana DNA methylation uncovers an interdependence between methylation and transcription. Nat Genet. 2007;39(1):61-9.

71. Bratzel F, Lopez-Torrejon G, Koch M, Del Pozo JC, Calonje M. Keeping cell identity in Arabidopsis requires PRC1 RING-finger homologs that catalyze H2A monoubiquitination. Curr Biol. 2010;20(20):1853-9.

72. Hennig L, Derkacheva M. Diversity of Polycomb group complexes in plants: same rules, different players? Trends Genet. 2009;25(9):414-23.

73. Huang G, Li T, Li X, Tan D, Jiang Z, Wei Y, et al. Comparative transcriptome analysis of climacteric fruit of Chinese pear (Pyrus ussuriensis) reveals new insights into fruit ripening. Plos One. 2014;9(9):e107562.

74. Garg R, Kumari R, Tiwari S, Goyal S. Genomic survey, gene expression analysis and structural modeling suggest diverse roles of DNA methyltransferases in legumes. Plos One. 2014;9(2):e88947.

75. Feng S, Cokus SJ, Zhang X, Chen PY, Bostick M, Goll MG, et al. Conservation and divergence of methylation patterning in plants and animals. Proc Nat Acad Sci U S A. 2010;107(19):8689-94.

76. Zemach A, Li Y, Wayburn B, Ben-Meir H, Kiss V, Avivi Y, et al. DDM1 binds Arabidopsis methyl-CpG binding domain proteins and affects their subnuclear localization. Plant Cell. 2005;17(5):1549-58.

77. Peng M, Cui Y, Bi YM, Rothstein SJ. AtMBD9: a protein with a methyl-CpGbinding domain regulates flowering time and shoot branching in Arabidopsis. Plant J. 2006;46(2):282-96. 
78. Yaish MW, Peng M, Rothstein SJ. AtMBD9 modulates Arabidopsis development through the dual epigenetic pathways of DNA methylation and histone acetylation. Plant J. 2009;59(1):123-35.

79. Vongs A, Kakutani T, Martienssen RA, Richards EJ. Arabidopsis thaliana DNA methylation mutants. Science. 1993;260(5116):1926-8.

80. Gendrel AV, Lippman Z, Yordan C, Colot V, Martienssen RA. Dependence of heterochromatic histone $\mathrm{H} 3$ methylation patterns on the Arabidopsis gene DDM1. Science. 2002;297(5588):1871-3.

81. Higo H, Tahir M, Takashima K, Miura A, Watanabe K, Tagiri A, et al. DDM1 (decrease in DNA methylation) genes in rice (Oryza sativa). Mol Gen Genomics. 2012;287(10):785-92.

82. Pikaard CS, Mittelsten Scheid O. Epigenetic regulation in plants. Cold Spring Harb Perspect Biol. 2014;6(12):a019315.

83. Deal RB, Topp CN, McKinney EC, Meagher RB. Repression of flowering in Arabidopsis requires activation of FLOWERING LOCUS C expression by the histone variant H2A.Z. Plant Cell. 2007;19(1):74-83.

84. Carbonell-Bejerano P, Rodriguez V, Royo C, Hernaiz S, Moro-Gonzalez LC, Torres-Vinals $M$, et al. Circadian oscillatory transcriptional programs in grapevine ripening fruits. BMC Plant Biol. 2014;14:78.

85. Lai AG, Doherty CJ, Mueller-Roeber B, Kay SA, Schippers JH, Dijkwel PP. CIRCADIAN CLOCK-ASSOCIATED 1 regulates ROS homeostasis and oxidative stress responses. Proc Natl Acad Sci U S A. 2012;109(42):17129-34.

86. Wong DC, Lopez Gutierrez R, Gambetta GA, Castellarin SD: Genome-wide analysis of cis-regulatory element structure and discovery of motif-driven gene co-expression networks in grapevine. DNA Res. 2017. doi:10.1093/ dnares/dsw061.

87. Millar AJ. The intracellular dynamics of circadian clocks reach for the light of ecology and evolution. Annu Rev Plant Biol. 2016;67:595-618.

88. Johansson M, Staiger D. Time to flower: interplay between photoperiod and the circadian clock. J Exp Bot. 2015;66(3):719-30.

89. Harmer SL, Hogenesch JB, Straume M, Chang HS, Han B, Zhu T, et al. Orchestrated transcription of key pathways in Arabidopsis by the circadian clock. Science. 2000;290(5499):2110-3

90. Fustin JM, Doi M, Yamaguchi Y, Hida H, Nishimura S, Yoshida M, et al. RNAmethylation-dependent RNA processing controls the speed of the circadian clock. Cell. 2013;155(4):793-806

91. Lokody I. Gene regulation: RNA methylation regulates the circadian clock. Nat Rev Genet. 2014;15(1):3.

92. Seo PJ, Mas P. Multiple layers of posttranslational regulation refine circadian clock activity in Arabidopsis. Plant Cell. 2014;26(1):79-87.

93. Malapeira J, Khaitova LC, Mas P. Ordered changes in histone modifications at the core of the Arabidopsis circadian clock. Proc Natl Acad Sci U S A. 2012;109(52):21540-5.

94. Webb AA, Satake A. Understanding circadian regulation of carbohydrate metabolism in Arabidopsis using mathematical models. Plant Cell Physiol. 2015;56(4):586-93.

95. Haydon MJ, Mielczarek O, Robertson FC, Hubbard KE, Webb AA. Photosynthetic entrainment of the Arabidopsis thaliana circadian clock. Nature. 2013:502(7473):689-92.

96. Houel C, Chatbanyong R, Doligez A, Rienth M, Foria S, Luchaire N, et al. Identification of stable QTLs for vegetative and reproductive traits in the microvine (Vitis vinifera L.) using the $18 \mathrm{~K}$ Infinium chip. BMC Plant Biol. 2015;15:205.

97. Martinez-Luscher J, Morales F, Sanchez-Diaz M, Delrot S, Aguirreolea J, Gomes $\mathrm{E}$, et al. Climate change conditions (elevated CO2 and temperature) and UV-B radiation affect grapevine (Vitis vinifera $\mathrm{cV}$. Tempranillo) leaf carbon assimilation, altering fruit ripening rates. Plant Sci. 2015;236:168-76.

98. Patel RK, Jain M. NGS QC toolkit: a toolkit for quality control of next generation sequencing data. Plos One. 2012;7(2):e30619.

99. Kersey PJ, Allen JE, Armean I, Boddu S, Bolt BJ, Carvalho-Silva D, et al. Ensembl genomes 2016: more genomes, more complexity. Nucleic Acids Res. 2016:44(D1):D574-80.

100. Dommes J, Vandewalle C. Polysome formation and incorporation of new Ribosomes into Polysomes during germination of the embryonic Axis of maize. Physiol Plant. 1990;79(2):289-96.

101. Li H, Handsaker B, Wysoker A, Fennell T, Ruan J, Homer N, et al. The sequence alignment/map format and SAMtools. Bioinformatics. 2009; 25(16):2078-9.

102. Anders S, Pyl PT, Huber W. HTSeq - a python framework to work with high-throughput sequencing data. Bioinformatics. 2014;31(2):166-9.
103. Robinson MD, McCarthy DJ. Smyth GK: edgeR: a Bioconductor package for differential expression analysis of digital gene expression data. Bioinformatics. 2010;26(1):139-40.

104. Benjamini Y, Hochberg Y. Controlling the false discovery rate: a practical and powerful approach to multiple testing. J Roy Stat soc ser B (Stat Method). 1995;57:289-300.

105. Love Ml, Huber W, Anders S. Moderated estimation of fold change and dispersion for RNA-seq data with DESeq2. Genome Biol. 2014;15(12):1-21.

106. Monaco MK, Stein J, Naithani S, Wei S, Dharmawardhana P, Kumari S, et al. Gramene 2013: comparative plant genomics resources. Nucleic Acids Res. 2014:42(Database issue):D1193-9.

107. Grimplet J, Van HJ, Carbonell-Bejerano P, Diaz-Riquelme J, Dickerson J, Fennell $\mathrm{A}$, et al. Comparative analysis of grapevine whole-genome gene predictions, functional annotation, categorization and integration of the predicted gene sequences. BMC Res Notes. 2012;5:213.

108. Kinsella RJ, Kahari A, Haider S, Zamora J, Proctor G, Spudich G, et al. Ensembl BioMarts: a hub for data retrieval across taxonomic space. Database (Oxford). 2011;2011:bar030.

109. Maere S, Heymans K, Kuiper M. BiNGO: a Cytoscape plugin to assess overrepresentation of gene ontology categories in biological networks. Bioinformatics. 2005;21(16):3448-9.

110. Leinonen $R$, Sugawara $H$, Shumway M, International Nucleotide Sequence Database C. The sequence read archive. Nucleic Acids Res. 2011; 39(Database issue):D19-21.

\section{Submit your next manuscript to BioMed Central and we will help you at every step:}

- We accept pre-submission inquiries

- Our selector tool helps you to find the most relevant journal

- We provide round the clock customer support

- Convenient online submission

- Thorough peer review

- Inclusion in PubMed and all major indexing services

- Maximum visibility for your research

Submit your manuscript at www.biomedcentral.com/submit 